\title{
Age-dependent Lamin remodeling induces cardiac dysfunction via dysregulation of cardiac transcriptional programs
}

\section{Natalie Kirkland}

University of California, San Diego

\section{Alexander Whitehead}

University of California, San Diego

James Hocker

University of California, San Diego

Pranjali Beri

University of California, San Diego

\section{Geo Vogler}

Sanford Burnham Prebsy Medical Discovery Institute

Bill Hum

Sanford Burnham Prebsy Medical Discovery Institute

\section{Mingyi Wang}

National Institute on Aging https://orcid.org/0000-0001-6412-369X

\section{Edward Lakatta}

National Institute on Aging

\section{Bing Ren}

University of California, San Diego

Rolf Bodmer

Sanford Burnham Prebys Medical Discovery Institute

Adam Engler ( $\sim$ aengler@ucsd.edu )

University of California, San Diego https://orcid.org/0000-0003-1642-5380

\section{Article}

Keywords: Sarcomere, transcription factor, nuclear lamina, lifespan, nuclear stiffness, chromatin accessibility

Posted Date: November 11th, 2021

DOI: https://doi.org/10.21203/rs.3.rs-1021378/v1 
License: (c) (i) This work is licensed under a Creative Commons Attribution 4.0 International License. Read Full License 
1 Age-dependent Lamin remodeling induces cardiac dysfunction via 2 dysregulation of cardiac transcriptional programs

3 (Author names redacted)

5 MANUSCRIPT INFORMATION

6 Abstract Count: 150 words

7 Main Text Character Count: 41,264 (including spaces and figure legends but excluding Methods

8 text, supplemental item legends, and References section)

9 Figure Count: 7

10 Supplemental Figure Count: 6

11 Supplemental Table Count: 10

12 Keywords: Sarcomere, transcription factor, nuclear lamina, lifespan, nuclear stiffness, chromatin

13 accessibility

14

15 RUNNING TITLE

16 Age-associated Nuclear Remodeling Drives Cardiac Dysfunction 


\section{Abstract}

18 As we age, structural changes contribute to progressive decline in organ function, which in the

19 heart acts through poorly characterized mechanisms. Utilizing the rapidly aging fruit fly model with its significant homology to the human cardiac proteome, we found that cardiomyocytes exhibit progressive loss of Lamin C (mammalian Lamin A/C homologue) with age. Unlike other tissues and laminopathies, we observe decreasing nuclear size, while nuclear stiffness increases. Premature genetic reduction of Lamin C phenocopies aging's effects on the nucleus, and subsequently decreases heart contractility and sarcomere organization. Surprisingly, Lamin $\mathrm{C}$ reduction downregulates myogenic transcription factors and cytoskeletal regulators, possibly via reduced chromatin accessibility. Subsequently, we find an adult-specific role for cardiac transcription factors and show that maintenance of Lamin $C$ sustains their expression and prevents age-dependent cardiac decline. Our findings are conserved in aged non-human primates and mice, demonstrating age-dependent nuclear remodeling is a major mechanism contributing to cardiac dysfunction.

\section{Introduction}

With aging comes a progressive decline in organ function ${ }^{1,2}$, but age-related decline in heart performance is especially critical as cardiovascular disease is the leading cause of mortality worldwide ${ }^{3}$. Aging results in the progressive loss of structural organization ${ }^{4,5}$, which can limit contractility ${ }^{1,6}$ and result in heart failure ${ }^{7}$. High prevalence of age-related cardiac dysfunction may in part be because cardiomyocyte renewal is limited ${ }^{8}$ and therefore, maintenance of cardiac function over time must rely on compensatory mechanisms; these are multifaceted but tightly linked to the integrity of key structural elements, e.g., intercalated discs, sarcomeres, and extracellular matrix. Reducing force on cardiomyocytes (CMs) or compensating with transgenic overexpression of intercalated disc proteins can partially reverse heart dysfunction, typically by restoring structural organization and gene expression ${ }^{6,7}$. Since physical forces transduced to the nucleus can impact chromatin organization and induce changes in gene expression ${ }^{9-11}$, nuclear remodeling may similarly be a mechanism of age-associated cardiac dysfunction.

Structural changes in the nucleus are primarily governed by the nuclear lamina, an intermediate 
cytoskeleton ${ }^{12,13}$ via the linker of the cytoskeleton (LINC) complex ${ }^{9-11}$ as well as to chromatin ${ }^{14,15}$

49 via lamina associated domains $(\mathrm{LADs})^{16}$. Along with the perinuclear cytoskeleton ${ }^{12,13}$ and 50 chromatin ${ }^{14,15}$, the nuclear lamina regulates nuclear properties, including stiffness, size and 51 shape ${ }^{17-26}$. In mechanically active tissues, Lamin mutations give rise to muscular dystrophy ${ }^{27,28}$ 52 and cardiomyopathies ${ }^{29}$, which also manifest in premature aging syndromes, e.g., Hutchinson Gilford Progeria (HGPS) ${ }^{30}$. Lamin mutations cause dysmorphic nuclei, epigenetic dysregulation and DNA damage ${ }^{31-34}$. However, changes in nuclear shape, which are conserved from invertebrates $^{35,36}$ to humans ${ }^{37}$, have been observed upon aging in the absence of Lamin mutations and accompany loss of heterochromatin ${ }^{37,38}$ and accumulation of DNA damage ${ }^{37}$. In some cases, Progerin (truncated Lamin A) has been identified in aging skin ${ }^{39}$ and dilated cardiomyopathy ${ }^{40}$ in the absence of mutations. Furthermore, Lamins decrease in expression with age in some tissues ${ }^{41-43}$, with loss of Lamin B being a well-known aging marker ${ }^{42}$ that may decrease cardiomyocyte regenerative capacity and increase ploidy ${ }^{44}$. Lamin A and C (Lamin A/C, two splice variants of the Imna gene) are the dominant adult cardiac Lamins, and age-associated reduction has been observed in mouse cardiomyocytes ${ }^{41}$, but a role in heart function and cardiac aging is unknown. Insights from Lamin A haploinsufficient mutant mice suggest Lamin reduction is as detrimental to heart function as progerin mutants; mice develop dilated cardiomyopathy via loss of sarcomere-nuclear coupling, show defective nuclear transport and fail to activate compensatory hypertrophic pathways ${ }^{45}$. Thus, age-associated nuclear remodeling could be a major mechanism contributing to organ dysfunction, yet mechanisms contributing to agedependent nuclear remodeling and how it affects tissue function remain elusive.

To investigate a role for age-dependent nuclear remodeling in regulating heart function, we primarily employ the invertebrate Drosophila melanogaster. Drosophila are rapidly aging, possess a simple but highly conserved heart ${ }^{46}$, and importantly, demonstrate age-depended cardiac decline ${ }^{5,47}$. We identified age-dependent remodeling unique to CM nuclei, which is strongly influenced by an age-dependent reduction of Lamin $\mathrm{C}$ (LamC), the fly homologue to mammalian Lamin A/C. Genetic reduction of LamC in young flies phenocopies age-associated nuclear stiffening, decreased heart contractility and sarcomere disorganization, and ultimately shortens lifespan. We show that LamC loss decreases expression of cardiomyocyte transcription 78 factors, as well as cytoskeletal regulators, likely by reducing their chromatin accessibility. 
79 Premature reduction of CM transcription factors partially phenocopies age-dependent loss of

80 heart function, while reinstating LamC levels in adult flies prevents transcription factor loss and 81 improves heart function. CM age-associated nuclear shrinkage is conserved from flies to non82 human primates and therefore presents nuclear remodeling as a major mechanism contributing 83 to age-related organ dysfunction.

\section{Results}

\section{Cardiomyocyte nuclei remodel during aging}

To understand whether age-associated nuclear remodeling influences heart function, we first sought to characterize how nuclear properties change upon aging in the Drosophila heart. Using two wildtype strains ( $w^{1118}$ and $y w$ ), we measured nuclear size and shape at 1, 3 and 5 weeks post-eclosure for surgically exposed hearts and specifically the A2-A3 region (Fig. 1A). Our highthroughput two-dimensional segmentation approach showed that common to both strains, $\mathrm{CM}$ nuclei decrease in cross-sectional area and became more circular upon aging (Fig. 1B and S1AC), which is contrary to long-standing observations in other cell types, e.g., skeletal muscle nucle ${ }^{36}$ and fibroblasts ${ }^{37}$. To exclude that our observations were an artifact of our protocol, we segmented nuclei from the syncytial ventral muscle that overlays the $\mathrm{CM}$ pairs within the same confocal images (Fig. 1A). Here, we found that ventral muscle nuclei increase in size upon aging, suggesting the reduction in nuclear size is CM specific (Fig. S1D). Nuclear atrophy is conserved in three-dimensions, as we found that CM nuclear volume also decreases with age in $w^{1118}$ flies (Fig. 1C and S1E). Since morphology and mechanics are often linked, we measured nuclear stiffness at 1 and 5 weeks of age using atomic force microscopy (AFM). CM nuclei, selected based on Hand-promoter specific nuclear GFP expression and size (smaller than the pericardial nuclei), were more than 2-fold stiffer in aged flies (Fig. 1D). Together, our results show that CM nuclei become smaller, more circular, and stiffer with age.

\section{Lamin RNA and protein levels decrease in cardiomyocytes during aging}

To identify candidate genes that may regulate age-associated nuclear remodeling, we performed bulk RNA sequencing on isolated Drosophila heart tubes to identify candidate genes that may regulate age-associated nuclear remodeling (Fig. 2A; Table S1). Approximately 1,487 differentially expressed genes (DEGs; $-1.25>$ FC $>1.25$, p-adj < 0.05 ) were identified and based 
110 on gene ontology (GO) analysis represented terms primarily related to the cytoskeleton and 111 sarcomere, ECM and adhesion, and chromatin regulation and nuclear envelope (Fig. 2B; Table 112 S2). Many DEGs in this latter ontology (Fig. S2A) are common to age-related terms, e.g., DNA 113 damage, repair, and histone regulation (Fig. S2B). Interestingly, several nuclear envelope genes 114 were downregulated including Lamin C (LamC) and two homologues of Nesprin, LINC complex 115 proteins, Klarischt (Klar) and Msp300 (Fig. 2C). Utilizing in situ Hybridization Chain Reaction $116(\mathrm{HCR})^{50}$ to visualize mRNA transcripts and confirm transcriptome analyses specifically in CMs, 117 we found that LamC mRNA expression indeed decreases upon aging as did Lamin B (LamB) 118 transcripts (Fig. S2C-D) consistent with other aging systems ${ }^{51}$. Other cell types present in the 119 heart may explain the absence of differential expression for LamB in bulk RNA sequencing (Fig. 120 1A). Subsequently, we verified via corrected total nuclear fluorescence (CTNF) that size121 normalized expression of LamB and LamC decreased upon aging (Fig. 2D and S2E). However, 122 unlike in Progeria and aged donor fibroblasts where Lamin $A / C$ relocates from nucleoplasm to 123 nuclear envelope ${ }^{37}$, Lamins did not show a redistribution within aged nuclei (Fig. S2E).

Cardiomyocyte Lamin C reduction phenocopies age-related nuclear and cytoskeletal remodeling, and shortens lifespan

Strong evidence from patients with laminopathies ${ }^{23,37}$ suggest that Lamins regulate cell function and contribute to heart dysfunction ${ }^{49}$. However, evidence does not suggest what effects, if any, there might be in aged hearts. To determine the effect of age-associated Lamin downregulation on CM function, we employed and verified cardiac-targeted RNAi for LamB and LamC through development relative to their background controls (attp40 for LamB RNAi and attp2 with Luciferase control RNAi for LamC RNAi; Fig. 3A and S3A-B). Using the non-targeted Lamin to mark nuclear lamina, we observed that LamC and LamB reduction decreased nuclear area and perimeter but only LamB RNAi increased circularity at 1 week of age (Fig. 3B and S3C). However, the effect size was age-dependent because controls undergoing age-associated remodeling more closely mirrored RNAi effects after 4 weeks of adulthood (Fig. S3D). These distinct effects on nuclear morphology indicate that LamC and LamB may dissimilarly influence nuclear properties. Indeed, nuclei extracted from LamC RNAi hearts at 1 week were stiffer than agematched controls and mimicked aged controls, while LamB RNAi hearts were softer than controls and did not phenocopy aging (Fig. 3C). 
Along with nuclear stiffness, we found differential effects on heart function upon LamB or LamC reduction. Surgically exposed hearts from 1 week and 4 week-old adults were subjected to live, high-speed imaging ${ }^{52}$. We observed that LamC RNAi hearts decreased in fractional shortening, i.e., the difference of systolic and diastolic heart diameters divided by the diastolic diameter, relative to age-matched controls (Fig. S3E-F). Conversely, LamB RNAi hearts only exhibited age-associated diminished contractility (Fig. 3D-E). Since hearts with reduced LamC were less contractile, we examined how organized the sarcomeres, i.e., the contractile unit of CMs, were to assess whether organization might account for reduced fractional shortening. Using an automated, unbiased Fourier transform analytic ${ }^{53}$, we found a significant decrease in sarcomere organization for LamC RNAi hearts relative to age-matched controls, which phenocopies 4-week adult heart organization. Consistent with functional data, LamB RNAi hearts only exhibited age-associated diminished organization (Fig. 3F-G). As heart function is tightly linked to survival in Drosophila, we observed that only LamC RNAi flies had a shortened lifespan (Fig. $3 \mathrm{H}$ ). These results suggest that LamC loss during aging contributes to heart dysfunction via sarcomere disorganization.

\section{Aging and Lamin C reduction lead to changes in chromatin accessibility and influence} mesoderm transcription factor loci

While heart dysfunction may occur via sarcomere disorganization, conserved upstream mechanisms for LamC RNAi and natural aging are unknown. Given Lamins' role in anchoring chromatin and their link to cardiomyopathies ${ }^{54}$, we hypothesized that cardiac dysfunction induced by Lamin deficits are mediated by changes in age-associated chromatin organization. Assay for Transposase-Accessible Chromatin sequencing (ATAC-Seq) was performed on isolated heart nuclei, and we verified detection of accessibility peaks mapping to Drosophila heart-specific and enriched genes Hand and tinman (tin) and sarcomere genes Tropomyosin and Mhc (Fig. 4A). Subsequently, we compared differentially accessible regions (DARs) for 1and 5-week wildtype $\left(w^{1118}\right)$, LamC RNAi and LamB RNAi hearts $(-1.25>$ FC > 1.25, p-adj < 0.1). There were more DARs with aging compared to RNAi hearts (Fig. 4B; Table S3), likely because aging impacts all cardiac-related cells whereas the RNAi was expressed only in CMs, which are a subset of all cells present in the heart tube (Fig. 1A). Surprisingly with less LamC, hearts had more DARs that were less accessible vs. more accessible, an imbalance also 
172 observed in aging hearts (67.5\% and $55.9 \%$, respectively). Conversely, LamB RNAi had fewer 173 DARs overall and fewer that were less accessible (38.0\%). Thus, while nuclei get smaller and 174 stiffer in aged and LamC RNAi hearts, there are also changes in chromatin accessibilty.

175 These data could suggest that $\operatorname{LamC}$ reduction might have effects on specific chromatin domains during aging, thus we asked to what extent the same genes were affected in the same direction for both aging and LamC RNAi. Analysis of DARs common to both datasets indicated that $68 \%$ of DARs were co-regulated, with more than half being less accessible (Fig. 4C; Table S4). Conversely, more DARs shared between LamB RNAi and aging were mutually more accessible. These results indicate that $\operatorname{Lam} C$ and $\operatorname{LamB}$ may differentially contribute to changes observed during aging, with $\operatorname{LamC}$ reduction conferring a decrease in accessibility associated with a decline in heart performance.

To better understand how co-regulated DARs might contribute to loss of function, we identified the ontological terms associated with the less accessible regions. Mutually less accessible genes revealed terms for contractile fibers and cell cortex in addition to differentiation, development, and morphogenesis (Fig. 4D; Table S5). Interestingly, the most highly significant terms' contributing genes included the Snail-type transcription factor Escargot and the heart188 specific transcription factor Hand (Fig. 4E), which do not change in accessibility for LamB RNAi (Fig. 4F). Hand is required for invertebrate and vertebrate CM specification ${ }^{55}$, and therefore may function beyond development to maintain cardiac programs. If reduced chromatin accessibility

191 leads to protein loss upon aging, it is possible that downstream cardiac expression could be 192 dysregulated.

Cardiomyocyte Lamin C loss exhibits a premature aging expression profile and decreases cardiomyocyte transcription factors

196 To assess whether altered chromatin accessibility might lead to transcriptional dysregulation, 197 and if other cardiac specific transcription factors were affected, we performed bulk-RNA 198 sequencing for aged and LamC RNAi hearts. We observed 344 differentially expressed genes 199 (DEGs) with heart-specific LamC loss and 1,998 DEGs in aged attp2 background flies (-1.25 > 200 FC > 1.25, padj < 0.05; Fig. 5A, Table S6-7) from which 688 DEGs were co-regulated in the 201 original $w^{1118}$ control aged hearts (Fig. S4A-C; Table S8). We then identified mutually significant 202 DEGs from LamC RNAi and aged hearts and observed that 111 DEGs were conserved in both 
203 conditions (Fig. 5B; Table S9). Mutually conserved genes presented biological process terms 204 related to aging (red, Fig. 5C; Table S9), suggesting that LamC loss creates differential gene 205 expression similar to natural aging. As validation, we also observed terms previously identified 206 from ATAC-Seq, including anatomical structure development and morphogenesis (blue, Fig. 5C), 207 in which CM transcription factors tin and H15 were downregulated (Fig. 5D). HCR validated CM 208 specificity of tin, H15, and Hand and showed that all three were reduced in both aged and LamC 209 RNAi hearts (Fig. 5E). Conversely for LamB RNAi, hearts showed only an aging phenotype and 210 no transgenic effect (Fig. 5E and S4D-E). Our results thus far show that LamC loss occurs with 211 age, makes nuclei smaller and stiffer, decreases CM transcription factor accessibility and 212 expression and then disrupts sarcomeres to cause contractile dysfunction. However, our results 213 do not yet establish if loss of a myogenic program is critical for adult myocyte function.

Adult-onset myogenic transcription factor loss induces heart dysfunction while LamC preserves heart function

The importance of myogenic transcription factors is highlighted by significant sarcomere defects present when any one factor is silenced throughout development (Fig. S5A). This begs the question of whether CM transcription factor loss in adulthood, due to age-associated LamC loss, could influence heart function. To reduce expression only in the adult fly and assess whether their loss phenocopies LamC reduction, flies possessing the temperature sensitive suppressor of Gal4, TubGal80's, and heart specific drive Hand-Gal4 were used (Fig. 6A). Within 24 hours of eclosure, adult flies were maintained at the permissive $\left(18^{\circ} \mathrm{C}\right)$ or shifted to the non-permissive temperature $\left(29^{\circ} \mathrm{C}\right)$, and after 2 weeks their heart function assessed. Live heart imaging showed that loss of each transcription factor only in adulthood still caused a significant decrease in

226 fractional shortening compared to control backgrounds which exhibit a slight, but insignificant reduction in fractional shortening due to relative differences in aging between flies maintained at $18^{\circ} \mathrm{C}$ verses $29^{\circ} \mathrm{C}$ (Fig. 6B-C and S5B).

Conversely, we asked if adult-onset LamC overexpression could preserve myogenic factor expression and function with age. When LamC expression is induced at $29^{\circ} \mathrm{C}$, we observed nuclear size was consistent with $18^{\circ} \mathrm{C}$ flies, in contrast to GFP overexpression controls 232 that showed an expected age-dependent reduction in nuclear size (Fig. 6D and S5C). LamC protein levels did not significantly decrease, in contrast to GFP overexpression controls (Fig. 
234 S5D), which corresponded to an increase in LamC transcript levels only at $29^{\circ} \mathrm{C}$ for LamC OE 235 hearts (Fig. S5E). Importantly, with additional LamC in older flies, fractional shortening was 236 preserved (Fig. 6E and S5F), as well as CM specific expression of myogenic transcript factors 237 tin, H15, and Hand (Fig. 6F-G). Together, our results establish that adult loss of myogenic 238 programs is mediated by age-associated LamC loss and their chromatin remodeling, which subsequently reduces adult cardiomyocyte function (Fig. 7A).

Nuclear Remodeling and Adult-onset Transcription Factor Loss is Conserved in Mice and Non-human Primates

Despite physiological differences between tubular and chambered hearts, there is surprising overlap between the Drosophila and human cardiac proteomes ${ }^{46}$. We therefore sought to assess whether similar structural and transcriptional changes are conserved from the fly heart to the mammalian heart ${ }^{41}$. We observed in both mouse and monkey heart sections that nuclear size decreased and circularity increased upon aging, as we found in the fly heart tube (Fig. 7B-C). Furthermore, immunofluorescence staining of the mouse heart sections confirmed reduction of Lamin A/C (Fig. 7B), consistent with Drosophila. Subsequently, we found mammalian homologues of fly transcription factors, Hand1, Nkx2.5 (homologue of tin), and Tbx20 (homologue of H15) significantly decreased expression in aging mice hearts (Fig. 7D and S6AB) and Hand1, Hand2 and Nkx2.5 significantly decreased in expression in aging non-human primate rhesus macaque hearts (Fig. 7E and S6C-D), when normalized to at least one of three different, stable housekeeping genes. These data suggest that the functional decline attributed to cardiomyocyte transcription factor loss in flies could be a conserved mechanism, caused in part by physical remodeling of the nucleus.

\section{Discussion}

259 The role nuclear remodeling has in heart function during natural aging has thus far been largely unexplored. Here, we demonstrate that CM nuclear remodeling, i.e., age-related loss of nuclear lamins, is intimately linked with tissue-level dysfunction. Genetically inducing nuclear remodeling leads to reduction in heart contractility, sarcomere disorganization and shortens lifespan by mimicking transcriptional changes that occur in natural aging. Our findings suggest that transcriptional misregulation downstream of nuclear remodeling may occur due to altered 
265 chromatin accessibility and, strikingly, this represses CM fate transcription factors and 266 sarcomeric structural components. Importantly, we show that preserving "youthful" nuclear 267 properties, e.g., high Lamin expression and nuclear morphology, maintains CM transcription 268 factor expression and heart function. These changes are conserved in both mice and non-human 269 primates further demonstrating nuclear remodeling and myogenic transcriptional programs as 270 potential therapeutic targets for preserving heart function during aging.

271 Our observations of age-associated nuclear remodeling in Drosophila, mice and non272 human primates cardiomyocytes are in contrast to existing observations in C. elegans intestinal 273 cells $^{35}$, Drosophila skeletal muscle ${ }^{36}$, aged human fibroblasts ${ }^{37}$ and what is currently understood 274 for progeria-related laminopathies ${ }^{19,23,33,37}$. Rather than increasing in size and dysmorphia, we 275 observe that aging CM nuclei atrophy and become rounder. We also, for the first time to our 276 knowledge, demonstrate that CM nuclei stiffen upon aging in situ, an observation only seen 277 previously in cell culture for progeria cells and only after multiple rounds of passaging ${ }^{19}$. Further 278 supported by our assessment of non-cardiomyocyte ventral muscle nuclei that hypertrophy with 279 age within the heart tube, our findings suggest that cardiomyocytes have specific mechanisms mediating nuclear remodeling.

In the context of Drosophila CMs, we sought to understand how nuclear remodeling occurred upon aging and identified that nuclear lamins, LamC and LamB, in addition to nesprinrelated proteins Klar and Msp300, were downregulated upon aging. Consistent with our data, Lamin B has been previously reported to be downregulated with age ${ }^{43,51,56}$ possibly due to its role in senesence ${ }^{42}$, while a functional role for age-associated Lamin A/C reduction has not previously been explored. We found that genetically reducing LamC prematurely was sufficient to induce aging-like nuclear atrophy and increased circularity, but conversely, overexpression was required to change nuclear size in Xenopus and HeLa ${ }^{25}$. While A and B-type Lamins differentially contribute to nuclear mechanics ${ }^{20}$, we observed that reduction of A-type LamC increased CM nuclear stiffness despite cultured cells' nuclei soften with reduced Lamin A/C expression ${ }^{20,26}$. These differences could be accounted for by several hypotheses; First, Drosophila LamB and LamC could have differing functions compared to mammalian counterparts, although in other cell types, there is conservation between Drosophila and human Lamins ${ }^{57}$. Second, it is increasingly apparent that nuclei respond differently in $2 D$ and $3 D$ environments. In 2D cell culture, nuclear wrinkling indicates membrane laxity, whereas in 3D 
296 environments ${ }^{58,59}$, wrinkling is dependent on actin filaments intrusion into the perinuclear space 297 and wrinkling infers high membrane tension ${ }^{58}$. Third, cell- or developmental-specific differences 298 may result in alternative mechanics upon Lamin depletion. For example, Jevtic et al., show that 299 in differentiated Xenopus cells, very high levels of Lamins can in fact decrease nuclear size ${ }^{25}$. 300 Fourth, cell-specific LADs at the nuclear periphery show unique phenotypes upon Lamin A 301 mutations in hiPSC-derived CMs versus adipocytes and hepatocytes ${ }^{60}$. Thus, differential Lamin302 chromatin interactions could similarly contribute to altered mechanical regulation in aging 303 cardiomyocytes verses other cell types.

304 Given the linkage of nuclear lamina to sarcomeres via the LINC complex and chromatin 305 via LADs ${ }^{61}$ as well as the functional deficits we uncovered, our data provide some of the first 306 confirmation for a role for A-type Lamins in age-dependent regulation of heart function. Removal 307 of Lamins disrupts chromatin attachment to the nuclear periphery, higher-order chromatin 308 organization, and can influence gene expression ${ }^{62-66}$. These studies focus predominantly on 309 stem cell fate and maturation, yet our data now suggests differences in postmitotic tissues. We 310 identified that in both aging and LamC reduction, differentially accessible peaks were skewed 311 towards decreased accessibility, despite evidence that heterochromatin is lost in Lamin A/C 312 mutants and with aging ${ }^{23,31}$. Correspondingly, studies specifically disrupting LADs yield 313 conflicting results depending on cell origin; Chang et al., reported chromatin decompaction and 314 redistribution in breast cancer cells ${ }^{66}$, while Ulianov et al., found that topological-associated 315 domains decondensed but global chromatin density increased in embryonic-derived Drosophila 316 S2 cells $^{64}$. Similarly, maintaining lamina but disrupting chromatin attachment increased 317 chromatin compaction in C. elegans embryos ${ }^{65}$. Disrupting LADs can also show localized 318 alterations in accessibility, with recent work demonstrating Lamin B loss leads to repositioning 319 of disease causing loci away from the nuclear periphery in post-mitotic neurones ${ }^{67}$ and alter 320 repressive $\mathrm{H} 3 \mathrm{~K} 9 \mathrm{me} 3$ marks in $\mathrm{C}$. elegans ${ }^{68}$. These conflicting instances, along with our data, 321 suggest that accessibility both globally, and locally for specific loci, could be context specific, 322 and thus our data suggests that in the context of aging, reduced accessibility could be coupled 323 to dysfunction.

324 We show that ultimately, LamC-mediated nuclear remodeling appears to be a conserved 325 process in vertebrates that reduces the expression of cardiomyocyte transcription factors, e.g., 326 Hand/HAND1/2, Tin/NKX2-5 and H15/Tbx20. We observe that Hand specifically is less 
327 accessible with aging and LamC reduction. In Drosophila, the highly conserved Tin is an early 328 initiator of cardiogenesis and binds between Hand exons 3 and $4^{69}$, an intron we observe to have 329 reduced accessibility upon LamC reduction (Fig. 4F). Thus, reduced gene accessibility could 330 further downregulate Hand and downstream myogenic transcription. We predict reduced 331 chromatin accessibility might also account for the reduction of Tin/NKX2-5 and H15/Tbx20 with 332 age across flies, mice, and monkeys. Our findings provide a new Lamin-mediated interpretation 333 for previous observations of reduced NKX2-5 in aged, isolated mouse cardiomyocytes ${ }^{70}$ and 334 provides them with a role beyond development. We show in Drosophila that their adult-specific reduction gives rise to a marked reduction in heart function, supported by studies that find an adult-specific role for TBX20 when deleted in mice ${ }^{71-73}$. Consistent with these observations, CM transcription factors are misregulated in remodeling events leading to heart failure ${ }^{74}$, e.g., HAND is downregulated in rodent hypertrophy ${ }^{75}$ and in human cardiomyopathy ${ }^{76}$. Therefore, Laminmediated misregulation of myogenic transcriptional programs likely has a significant impact on mediating heart dysfunction during aging and may precede the development of heart failure. Since preserving LamC, and therefore nuclear morphology, maintained CM transcription factor expression and heart function despite aging in flies, our findings suggest nuclear lamina remodeling is a unique mechanism in age-related organ dysfunction. Furthermore, our work presents several avenues for investigating therapeutic interventions to increase health span into advanced age.

\section{Methods}

\section{Drosophila melanogaster}

349 Fly stocks were raised in non-crowded conditions on standard fly food medium consisting of yeast, cornstarch and molasses (10\% yeast, $12 \%$ sugar and $1.5 \%$ agar). Flies were raised at $25^{\circ} \mathrm{C}$ except for the temperature sensitive fly crosses (HandGal4, TubGal80ts; TubGal80ts, Fig. 6) which were raised at $18^{\circ} \mathrm{C}$ until eclosure, then $50 \%$ of eclosed flies were aged at $29^{\circ} \mathrm{C}$ and $50 \%$ at $18^{\circ} \mathrm{C}$. Freshly eclosed flies were collected and aged such that day of collection was day 1. Flies were transferred to fresh food every 2-3 days. Female flies were used for subsequent heart analysis to ensure consistent heart morphology. The following fly lines were used from the Bloomington stock center: white-1118, $w^{1118}$, yellow-white yw, attp2; UAS-Luciferase (\#31603), UAS-LamC RNAi (\#31621), attp40 (\#36304), UAS-LamB RNAi (\#57501), UAS-Stinger-GFP 
(\#84277), UAS-tinman RNAi (\#50663), UAS-H15 RNAi (\#57415), UAS-Hand RNAi (\#28977). Hand ${ }^{4.2}$ Gal4 was acquired from Olsen Laboratory ${ }^{69}$ and modified by the Bodmer lab to make Hand ${ }^{4.2}$ Gal4, TubGal80ts; TubGal80ts. UAS-LamC was gifted by the Walrath laboratory.

\section{Mouse}

All mouse experiments were performed in according to the guidelines established by the Institutional Animal Care and Use Committee at the University of California San Diego. Use of aged C57BL/6 mice was approved by the University of California San Diego Institutional Animal Care and Use Committee under study \#S08172. All animals were provided with food and water ad libitum until the specific age time point at which point animals were euthanized by asphyxiation followed by cervical dislocation. The lower section of the left ventricle was removed from five young (5-month), three juvenile (9-month), four adult (14-month), and three aged (24month) old mice and snap-frozen in liquid nitrogen immediately after resection and stored at $80^{\circ} \mathrm{C}$. The remainder of the heart was washed in PBS before embedding in OCT for cryosectioning. OCT boats containing hearts were frozen on dry ice with methyl butane before storage at $-80^{\circ} \mathrm{C}$.

\section{Rhesus Macaque}

Ten adult male rhesus monkeys (ages: 8.87, 9.7, 10.66, 12.88, 14.12, 18.81, 19.59, 23.39, 24.73, and 25.48 years of age) were maintained at the NIA in accordance with NIH Institutional Animal Care and Use Committee protocol AG000238-07 (Effects of Aging on Experimental Atherosclerosis in Nonhuman Primates). Left ventricular samples from macaque where flashfrozen for qPCR analysis or formalin fixed, paraffin embedded and subsequently sectioned for immunofluorescence analysis.

\section{Fly heart dissection}

Flies were anaesthetized with FlyNap ${ }^{\circledR}$ (Carolina Biological Supply Co.) and dissected in artificial hemolymph that was oxygenated using aerators as previously described ${ }^{52}$. 


\section{Immunofluorescence and imaging}

388 Hearts dissected in oxygenated artificial hemolymph were relaxed using 10mM EGTA in 389 oxygenated artificial hemolymph and immediately fixed with $4 \%$ formaldehyde in the same EGTA 390 hemolymph solution for 20 minutes. The hearts were then rinsed $3 \times$ with phosphate buffered 391 saline (PBS) and washed $3 \times 10$ minutes with $0.5 \%$ Triton 100-X in PBS (PBST). The hearts 392 were then blocked with 1\% BSA in PBST (PBST-BSA) for 30 minutes. Primary antibodies were 393 prepared as indicated below in PBST-BSA and incubated overnight at $4^{\circ} \mathrm{C}$. PBST and PBST394 BSA washes were repeated and secondary antibody with DAPI and Phalloidin were prepared in 395

For imaging, the cuticle around the hearts was subsequently trimmed down to a small rectangle to prevent obstruction of the heart, then hearts were transferred to Fluormount ${ }^{\circ} \mathrm{G}$ slide mounting medium for antibody-based imaging or ProLong ${ }^{\text {TM }}$ Glass Mountant (Invitrogen) for HCR imaging. The A2-A3 region of the heart was imaged on a Zeiss LSM780 inverted confocal microscope with a 40X objective, $1 \times$ Zoom, $0.44 \mu \mathrm{m}$ depth resolution for nuclear imaging or $0.88 \mu \mathrm{m}$ for actinin and HCR imaging, and at a resolution of $2148 \times 1076 \mathrm{XY}$ pixels.

Mouse heart sections embedded in OCT were cryosectioned and stored at $-80^{\circ} \mathrm{C}$ prior to fixation and staining. Slides were directly fixed with $4 \%$ PFA in PBS for 20 minutes at $-20^{\circ} \mathrm{C}$ with regular agitation to prevent freezing. Slides were subsequently washed $3 \times 5$ minutes with PBS and permeabilized for 1 hour with 1\% PBS-Triton 100-X. Primary antibody was prepared in 10\% Fetal Bovine Serum (FBS) with PBS (anti-Lamin A/C 1:250, Cell Signalling 4C11) and incubated overnight at $4^{\circ} \mathrm{C}$. Slides were subsequently washed $3 \times 5$ minutes with PBS before applying secondary antibody (Donkey anti-mouse Alexa Fluor 488, ThermoFisher, A21202, 1:500) and DAPI (Sigma). Slides were then washed $3 \times 5$ minutes with PBST and then PBS. Finally, samples were prepared for imaging using ProLong ${ }^{\mathrm{TM}}$ Glass Antifade Mountant (Invitrogen). Samples were 
418 imaged on a Keyence All-in-One BZ-X Series Fluorescence Microscope, with a 60X objective, $4191 \times$ Zoom, $1 \mu \mathrm{m}$ depth resolution and 1920 x $1440 \mathrm{XY}$ pixel resolution.

421 Macaque heart sections were received from the NIA. For staining and imaging, slides were first 422 rehydrated using the following steps: $2 \times 10$ minutes with Xylene, 100\% ethanol, 95\% ethanol 423 (in DI water), 70\% ethanol and 50\% ethanol before rinsing with DI water. Slides were 424 subsequently immersed in PBS with $0.5 \%$ Triton X-100 for 30 minutes and incubated with DAPI 425 (Sigma) for 30 minutes, prior to $3 \times 5$ minute washes with PBST and $3 \times 5$ minutes with PBS. 426 Slides were prepared using ProLong ${ }^{\mathrm{TM}}$ Glass Antifade Mountant (Invitrogen) and imaged as 427 described for mouse heart sections.

\section{Fly nuclear morphology and intensity analysis}

430 For two-dimensional analysis of nuclear morphology, 3D stack images were acquired of the A2431 A3 region of the heart as described above in the (Methods: Immunofluorescence and Imaging). 432 The A2-A3 heart region possesses 3-4 cardiomyocyte pairs and therefore 6-8 total CM nuclei. 433 Using ImageJ, the CM nuclei were cropped from the larger heart image, within a $22.17^{2} \mu \mathrm{m} /$ $434224^{2}$ pixel box with the minimal number of $z$ slices to eliminate out-of-plane nuclei from the cuticle 435 or ventral muscle overlaying the CM nucleus. The cropped nuclei were then segmented by the 436 following function in a batch macro workflow:

//Setting the measurement parameters run("Set Measurements...", "area perimeter fit display redirect=None decimal=3");

//Define directory input $=$ " ";

output $="$ ";

//For batch analysis setBatchMode(true); list $=$ getFileList(input); for $(\mathrm{i}=0 ; \mathrm{i}<$ list.length; $\mathrm{i}++)$ 
action(input, output, list[i]);

setBatchMode(false);

478 The results were then saved and analyzed in excel. To calculate aspect ratio (AR) the minor axis 479 was divided by the major axis and to calculate the circularity the excel function 
$480 "=\left(4^{*} \mathrm{PI}()^{*} \mathrm{C} 2\right) / \mathrm{D} 2^{\wedge} 2^{\prime}$ was used. The data was presented, and the appropriate statistical tests 481 performed in prism.

483 For three-dimensional analysis of nuclear morphology, the FIJI 3D Mesh plugin ${ }^{80}$ was used on 484 the LamC channel of the cropped nuclei stacks. The parameters for seeding and expanding the 485 mesh were as follows: gamma, 200.0; pressure, 0.06; image weight, 0.05; beta 0.0; alpha, 1.0; 486 steric neighbors, 0.0; divisions, 3.0. Volume and surface area from exported results were copied 487 to prism for graphing and statistical testing.

489 The corrected total nuclear fluorescence (CTNF) was calculated using ImageJ. Cropped nuclear 490 stacks, a binary image of the Lamin channel and max projection image were generated as 491 described above in Nuclear Morphology Analysis. An ROI is generated from the binary and 492 overlaid on the max projection image and area and integrated density are measured for the 493 Lamin channel. decimal=3");

//Setting the measurement parameters

run("Set Measurements...", "area mean min integrated display redirect=None

//Define directory

//Call lists

PathMax = " ";

PathBinary = " "; 
536 Subsequently, to account for changes in nuclear size, the background is subtracted relative the 537 area. A clear region outside of the nucleus is selected from the Lamin channel and the mean 538 intensity measured. Then, the following equation is used to calculate the approximate protein 539 amount: Corrected Total Nuclear Fluorescence = Integrated Density $-($ Mean Intensity $\times$ Nuclear 540

//Open binary image

open(PathBinary+listBinary[i]);

//Set to the Lamin Channel

Stack.setChannel(3);

//Generate ROI and close binary

run("Analyze Particles...", "size=20.00-200.00 display add slice");

close();

//Open max projection image

open(PathMax+listMax[i]);

//Overlay ROI on max projection image

roiManager("Select", 0);

//Measure intensity of Lamin channel

Stack.setChannel(3);

run("Measure");

//Reset for next image in list

close();

roiManager("Delete");

\} Area). 


\section{Lamin localization}

543 A custom python code ${ }^{81}$ was modified to assess the intensity of Lamin at radially increasing

544 distances from the center of the nucleus to the periphery for the max projected images also 545 generated for nuclear morphology and intensity analysis (Figure S2F). The average mean 546 intensity measurement at periphery was then divided by the average mean intensity the center 547 to obtain the fold enrichment of Lamin at the periphery.

\section{Sarcomere organization}

550 Using ImageJ and confocal stack images of actinin stained hearts, the dorsal region of the A2551 A3 region was projected to isolate a planar region of sarcomeres and eliminate actinin-stained 552 sarcomeres from the ventral side of the CMs and ventral muscle. ROls with a single layer of 553 sarcomeres uninterrupted by non-CM cells were then cropped and saved. The isolated actinin 554 regions from A2-A3 region were then batch processed using a published matlab $\operatorname{code}^{53}$ which 555 uses a scanning Fourier transform to calculate organizational index. The input parameters 556 included a sarcomere length of $2.5-3.2 \mu \mathrm{m}$, a sarcomere directionality of $90^{\circ}$, a scanning 557 resolution of 16 and at the appropriate pixel to $\mu \mathrm{m}$ ratio.

\section{Lifespan assay}

560 To determine lifespan, virgin females were collected and up to 30 flies separated into each vial. 561 The flies were maintained at $25^{\circ} \mathrm{C}$ and transferred to fresh food every 2-3 days, when dead 562 flies were also counted.

563 speed digital imaging (142fps, 9300 EM-CCD cameras, Hamamatsu), a 10X water-immersion

567 lens and HClmageLive software (Hamamatsu). Using semi-automatic optical heart beat 568 analysis software $(\mathrm{SOHA})^{82}$, fractional shortening was calculated from the end diastolic diameter (EDD) and end systolic diameters (ESD): (FS = EDD-ESD/EDD).

\section{Nuclear extraction}


$57230-60$ dissected hearts were removed from their cuticle and transferred to $1 \mathrm{ml}$ of ice-cold Nuclei 573 EZ lysis buffer (Sigma-Alrdich Nuclei EZ Prep isolation kit) in a $1 \mathrm{ml}$ glass douncer. 20 loose 574 strokes followed by 10 minutes on ice and then 15 tight strokes aided dissociation of nuclei from 575 the hearts. The solution was transferred to a low-bind Eppendorf and centrifuged at $500 \times \mathrm{g}$ for 5765 minutes. The supernatant was removed, the nuclear pellet resuspended in fresh ice-cold 577 Nuclei EZ lysis buffer and incubated on ice for 5 minutes. Spinning, removal of supernatant, 578 resuspension and incubation on ice was repeated once more. The samples were then 579 centrifuged once more at $500 \times \mathrm{g}$ before removing the supernatant and resuspending the pellet in PBS for nuclear AFM or Nuclei EZ storage buffer for ATAC Seq samples.

\section{Atomic Force Microscopy}

583 For atomic force microscopy, isolated nuclei in PBS were spun (500g, 3 minutes) on to $12 \mathrm{~mm}$ coverslips coated with Poly-D-Lysine $(1 \mu \mathrm{g} / \mu \mathrm{l}$ was used to coat coverslips for 5 minutes, then rinsed with purified water and left to dry overnight). Coverslips were transferred to a glass slide, secured with vacuum grease, and covered in a PBS droplet for AFM. Indentation experiments were performed on an MFP-3D Bio Atomic Force Microscope (Oxford Instruments) mounted in a Ti-U fluorescent inverted scope (Nikon Instruments, Melville, NY) and used Asylum Research 13, Igor Pro 6.34A software. Nanoworld PNP-TR tips were calibrated for their spring constant using the thermal noise method and used for probing isolated nuclei. A trigger force of $2 \mathrm{nN}$, an approach Velocity constant of at $2 \mu \mathrm{m} / \mathrm{s}$ and a force distance of $6 \mu \mathrm{m}$ were used to generate a force map with 12 points across $2 \mu \mathrm{m}^{2}$. Hand4.2-Gal4 was used to drive expression of GFP, and thus only GFP-positive nuclei were selected for indentation. The software was used to calculate the Young's modulus using the Hertz equation ${ }^{79}$. Any poor fits to the indentation curve were excluded. Then, the average Young's modulus was calculated from the force map.

\section{Bulk RNA sequencing}

598 For gene expression analysis, corresponding adult flies were dissected as previously described ${ }^{52}$ to expose the heart. Fat cells were carefully removed from either side of the length 600 of the heart. A minimum of 15 hearts were then pulled from the cuticle using fine forceps and

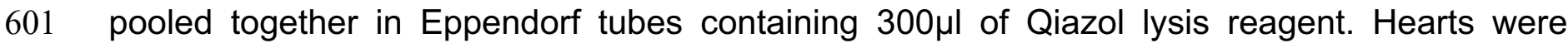


603 a further $400 \mu \mathrm{l}$ of Qiazol lysis reagent was added and the tube flash frozen in liquid nitrogen. 604 Samples were stored for up to 2 weeks at $-80^{\circ} \mathrm{C}$ until RNA extraction was performed. Total RNA 605 was then extracted and purified using the Qiagen miRNeasy Mini kit (Cat. No. 217004) as per 606 the protocol. The purified RNA was then processed by the Institute for Genomic Medicine at 607 University California San Diego. RNA integrity was analyzed using an Agilent Tape station 608 system and precise RNA concentration determined using a Qubit 2.0 Fluorometer. Libraries were 609 built using the Illumina TruSeq Stranded RNA, High Throughput Library Prep Kit and sequenced 610 on a NovaSeq 6000 for samples with RIN numbers at 9.0 and above.

612 RNA-Sequencing data was analyzed by ROSALIND® (https://rosalind.onramp.bio/), with a 613 HyperScale architecture developed by ROSALIND, Inc. (San Diego, CA). Reads were trimmed 614 using cutadapt ${ }^{83}$. Quality scores were assessed using FastQC ${ }^{84}$. Reads were aligned to the 615 Drosophila melanogaster genome build dm6 using STAR ${ }^{85}$. Individual sample reads were 616 quantified using HTseq ${ }^{86}$ and normalized via Relative Log Expression (RLE) using DESeq2 R 617 library ${ }^{87}$. Read Distribution percentages, violin plots, identity heatmaps, and sample MDS plots 618 were generated as part of the QC step using RSeQC ${ }^{88}$. DEseq2 was also used to calculate fold 619 changes and $p$-values and perform optional covariate correction. Clustering of genes for the final 620 heatmap of differentially expressed genes was done using the PAM (Partitioning Around 621 Medoids) method using the $\mathrm{fpc} R$ library ${ }^{89}$. Hypergeometric distribution was used to analyze the 622 enrichment of pathways, gene ontology, domain structure, and other ontologies. The topGO R 623 library ${ }^{90,}$, was used to determine local similarities and dependencies between $\mathrm{GO}$ terms in order 624 to perform Elim pruning correction. Several database sources were referenced for enrichment 625 analysis, including Interpro ${ }^{91}, \mathrm{NCBI}^{92} \mathrm{MSigDB}^{93,94}, \mathrm{REACTOME}^{95}$, WikiPathways ${ }^{96}$. Enrichment 626 was calculated relative to a set of background genes relevant for the experiment. Panther was 627 used to assess GO terms for gene lists generated in Rosalind.

629 Hybridization Chain Reaction (HCR)

630 Hearts were dissected as previously described ${ }^{52}$ to expose the heart in a $2.5 \mathrm{~mm}$ dish. The hearts 631 were relaxed with 10mM EGTA in oxygenated hemolymph and fixed with $4 \%$ formaldehyde in $6320.1 \%$ Tween 20, PBS for 20 minutes. Next, the hearts were washed $2 \times 5$ minutes with $0.1 \%$ 633 Tween 20, PBS. Then on ice, hearts were incubated, each for 5 minutes, with $25 \%, 50 \%, 75 \%$, 
$634100 \%, 75 \%, 50 \%$ and finally 25\% methanol in PBS. Hearts were then permeabilized for 2 hours 635 at room temperature with $1 \%$ Triton $100-\mathrm{X}$ in PBS. A second fixation was repeated at room 636 temperature and samples were washed $2 \times 5$ minutes with $0.1 \%$ Tween, PBS on ice. Then, a $63750 \%$ 0.1\% Tween 20, PBS; 50\% 5X SSCT (20XSSC, 10\% Tween 20, Ultrapure water) solution 638 was used to wash the samples for 5 minutes on ice and replaced by 5 X SSCT for a further 5 639 minutes. The cuticle with the heart attached was then trimmed down to a small rectangle and 640 carefully transferred to a 96 well plate well (each containing a maximum of 7 hearts). Within the 641 well, the hearts were incubated with probe hybridization buffer (Molecular Instruments) on ice 642 for 5 minutes, then the plate was transferred to $37^{\circ} \mathrm{C}$ for 30 minutes. $2 \mu \mathrm{l}$ of each probe designed 643 by Molecular Instruments was prepared in $200 \mu \mathrm{l}$ of probe hybridization buffer and incubated 644 overnight with the hearts at $37^{\circ} \mathrm{C}$. The following day, the samples were washed $4 \times 15$ minutes 645 with probe wash buffer (Molecular Instruments); $2 \times 5 \mathrm{XSST}$ and $1 \times 5$ minutes with 646 amplification buffer (Molecular Instruments). To prepare the hairpins for fluorescence 647 amplification, $2 \mu \mathrm{l}$ of corresponding h1 and h2 were heated to $95^{\circ} \mathrm{C}$ for 90 seconds and cooled in 648 the dark for 30 minutes. The cooled hairpins were then added to $100 \mu$ of amplification buffer 649 and incubated with the hearts overnight at room temperature in the dark. On the next day, while 650 maintained in the dark, the samples were washed $2 \times 5$ minutes with 5X SSCT; $2 \times 30$ minutes 651 with $5 \times$ SSCT; 1 x 5 minutes with $5 X$ SSCT and finally rinsed $3 \times$ with PBS. DAPI (1:250) was 652 added with the first 5 X SSCT 30-minute wash or stained subsequently for 15 minutes in PBST, 653 followed by $3 \times 10$-minute PBST washes and $3 \times$ PBS rinses. Samples were prepared and 654 imaged as described above (Methods section: Immunofluorescence and Imaging).

655

To quantify RNA expression levels, the processed hearts were imaged as described in the Immunofluorescence and Imaging section and then imported into ImageJ. For Hand, Tinman and $\mathrm{H} 15$ quantification, the $\mathrm{A} 2-\mathrm{A} 3$ heart region confocal stack was converted to a max projection, duplicated and then binarized. Using the max projected image as a guide, the cytoplasmic pockets surrounding the $\mathrm{CM}$ nuclei were then then traced, the ROI copied to the binary imaged 662 663 for particle analysis. As the segmentation was imperfect for transcripts very close together and to account for differences in pocket size, the \% area covered by the transcripts was used to assess statistical significance in Prism (Graphpad). 
665 As Lamin C and B are expressed in cells other than the CM nuclei, i.e., the ventral muscle nuclei 666 and the cuticle, the narrowest stacks were taken around the nuclear-cytoplasmic pocket to 667 eliminate interfering non-CM transcripts, and then the same analysis was conducted as for $\mathrm{H} 15$, 668 Tinman and $\mathrm{H} 15$. The macro is as follows:

669

670

671

672

673

674

675

676

677

678

679

680

681

682

683

684

685

686

687

688

689

690

691

692

693

694

695

//Generate max projection image of the confocal stack image

run("Z Project...", "projection=[Max Intensity]");

Stack.setDisplayMode("composite");

//Maintains a copy of the max projection image

run("Duplicate...", "duplicate");

//Binarize the max projection image

run("Subtract Background...", "rolling=5 stack");

run("Gaussian Blur...", "sigma=1 stack");

setAutoThreshold("Triangle dark");

setOption("BlackBackground", true);

run("Convert to Mask", "method=Triangle background=Dark calculate black");

run("Watershed", "stack");

\section{Bulk ATAC Sequencing}

ATAC-seq was performed on 2,000-5,000 nuclei per sample. Samples were permeabilized in cold nuclear permeabilization buffer ((0.2\% IGEPAL-CA630 (I8896, Sigma), 1 mM DTT (D9779, Sigma), Protease inhibitor (05056489001, Roche), and 5\% BSA (A7906, Sigma) in PBS (1001023, Thermo Fisher Scientific)) for 5 minutes on a rotator at $4^{\circ} \mathrm{C}$ followed by centrifugation for 5 min at $500 \mathrm{~g}$ at $4^{\circ} \mathrm{C}$. After decanting supernatant, the pellet was resuspended in cold tagmentation buffer ((33 mM Tris-acetate $(\mathrm{pH}=7.8)$ (BP-152, Thermo Fisher Scientific), $66 \mathrm{mM}$ K-acetate (P5708, Sigma), 11 mM Mg-acetate (M2545, Sigma), 16\% DMF (DX1730, EMD Millipore) in molecular biology grade water (46000-CM, Corning)) followed by incubation with Tagmentation enzyme (FC-121-1030; Illumina) at $37^{\circ} \mathrm{C}$ with shaking at $500 \mathrm{rpm}$ for $30 \mathrm{~min}$. Tagmented DNA was purified using MinElute PCR purification kit (28004, QIAGEN). The 
696 resulting libraries were amplified using NEBNext High-Fidelity 2X PCR Master Mix (M0541, 697 NEB) with primer extension at $72^{\circ} \mathrm{C}$ for 5 minutes, denaturation at $98^{\circ} \mathrm{C}$ for $30 \mathrm{~s}$, followed by 8 698 cycles of denaturation at $98^{\circ} \mathrm{C}$ for $10 \mathrm{~s}$, annealing at $63^{\circ} \mathrm{C}$ for $30 \mathrm{~s}$ and extension at $72^{\circ} \mathrm{C}$ for $60 \mathrm{~s}$. 699 After purification of amplified libraries using MinElute PCR purification kit (28004, QIAGEN), 700 double sided size selection was performed using SPRIselect beads (B23317, Beckman Coulter) 701 with $0.55 \mathrm{X}$ beads and $1.5 \mathrm{X}$ to sample volume.

702

Sample Processing from FASTQ - FASTQ files were submitted through the (redacted) 704 Epigenetics ATAC-seq pipeline (https://github.com/(redacted)), based on the ENCODE pipeline. 705 Briefly, reads were aligned using bowtie2, converted to uncompressed BAM files, sorted and 706 index using: bowtie2-X2000 --mm --local -1 \$fastq1 -2 \$fastq2 | samtools view -Su /dev/stdin | 707 samtools sort \& index > xxx.PE2SE.bam \&.bai 2> align.log. Poorly mapped, (<30 mapping 708 score), duplicate, multimapped, and mitochondrial reads were removed using samtools and 709 picard. Tn5 adapters were removed by truncating + end reads by 4 base pairs and - end reads 710 by 5 base pairs, and then written to final output BAMs.

712 Computational Analysis - BAM files were downloaded from (redacted) Center for Epigenomics, 713 sorted and indexed with samtools. Peakcalling was performed using MACS2 using the following commands: callpeak -f BAMPE -g dm - -q 0.01 --nomodel --shift -100 --extsize 200 --keep-dup all. MACS .xls output files and sorted BAMs were used to construct a Diffbind3.0.9 sample sheet 716 for each comparison: 1- week vs 5-week w1118 samples, wildtype vs LamB iR attp40 samples, 717 and wildtype vs LamC iR attp2 samples. Samples were read into R Studio using dba(), count 718 densities per peak were calculated using dba.count(), filtering out peaks with $<1$ read per sample 719 and a summit width of 100 (as recommended by the Diffbind3 vignette). Differential accessibility 720 was calculated using the EdgeR wrapper of dba.analyze(). BED files were generated for each 721 comparison using dba.report() and annotated using HOMER annotatePeaks.pl. Regions were 722 filtered based on a log2 fold change of 0.32 and FDR of $\leq 0.1$. Common features between 723 comparisons were isolated using dplyr's inner_join function of the "Nearest.Refseq" column 724 output of HOMER. Plots were generated using ggplot2 and ggrepel packages. Panther was used 725 to assess GO terms for gene lists. 


\section{Quantitative PCR for Monkey and Mouse left ventricle}

728 Total RNA was isolated from mouse and monkey frozen left ventricle sections by first, grinding 729 frozen tissue in a pestle and mortar with liquid nitrogen to ensure samples did not degrade.

730 Ground tissue was transferred to an Eppendorf and resuspended in 600ul of RLT lysis buffer 731 from the RNeasy mini RNA extraction kit (Qiagen). The suspension was then transferred to a 732 QIAshredder column and centrifuged at $<10,000$ rcf for 5 minutes for further homogenization. 733 The supernatant was collected and total RNA was extracted using the RNAeasy mini RNA 734 extraction kit (Qiagen) as per the protocol. RNA quality was assessed using an Agilent Tape station system. Poly $(A)^{+}$RNA was reverse transcribed using oligo(dT) reagent of the SuperScript IV First-Strand Synthesis kit (Thermofisher) and cDNA library generated using manufacturers protocol with a final RNase step. RT-qPCR was then performed in triplicate for each sample using SYBR Green PCR Master mix (Thermofisher) and the CFX96 hardware (Biorad). Each gene of interest was normalized to three housekeeping genes ${ }^{77,78}$ using the delta CT equation 2-(AvgCqGOI-AvgCqHK) . Primer sequences are shown in Table S10 and validated for specificity by melt temperature, and efficiency by DNA concentration titration are shown below.

\section{Statistical analysis}

Microsoft Excel 2011, Matlab 2020a, Python and Prism 9 Software were used to present data and conduct statistical analysis. The respective statistical tests and $\mathrm{n}$ numbers are described in the figure legends. For nuclear morphology and intensity analysis and HCR, 6-8 nuclei were cropped from the A2-A3 heart section and a minimum of 7 hearts were assessed. For RNA extraction, 15 hearts were collected per condition, and at least three biological replicated were acquired. For nuclear extraction 30-50 hearts were extracted per condition and 3-5 replicates were obtained. For SOHA live heart imaging, $>13$ hearts were imaged and analyzed. For actinin organization, $>14$ hearts were analyzed. For lifespan assays, more than 100 flies were recorded. The following statistical significance cut off was applied: n.s. $p>0.05,{ }^{*} p<0.05,{ }^{* *} p<0.01$, distributions. 


\section{Data and code availability}

758 Software to image fly hearts, analyze their contraction, and create kymographs, i.e., Semi759 automatic Optical Heartbeat Analysis, SOHA, is available at http://sohasoftware.com/index.html. 760 Python code to assess Lamin distribution is available at (redacted). Any ImageJ macros have 761 been included in Quantification and Statistical Analysis Section in Materials and Methods. RNA762 Seq and ATAC-Seq data is deposited at Gene Omnibus Express (GEO) Accession GSE 763 (redacted) and GSE (redacted), respectively.

\section{Acknowledgements}

767

\section{Author Contributions}

769 Redacted.

770

\section{Competing Interests}

772 The authors declare no competing interests.

773

\section{4}

\section{References}

775 1. Phillip, J. M., Aifuwa, I., Walston, J. \& Wirtz, D. The Mechanobiology of Aging. Annu Rev Biomed $776 \quad$ Eng 17, 113-141 (2015).

777 2. Gilbert, H. T. J. \& Swift, J. The consequences of ageing, progeroid syndromes and cellular 778 senescence on mechanotransduction and the nucleus. Exp Cell Res 378, 98-103 (2019).

779 3. CDC, N. Underlying Cause of Death 1999-2013 on CDC WONDER Online Database, released 780 2015. Data are from the Multiple Cause of Death Files, 1999-2013, as compiled from data provided by the 57 vital statistics jurisdictions through the Vital Statistics Cooperative . (2015).

4. Sessions, A. O. et al. Extracellular matrix downregulation in the Drosophila heart preserves contractile function and improves lifespan. Matrix Biol 62, 15-27 (2017).

5. Kaushik, G. et al. Vinculin network-mediated cytoskeletal remodeling regulates contractile function in the aging heart. Sci Transl Med 7, 292ra99 (2015).

6. Sessions, A. O. \& Engler, A. J. Mechanical Regulation of Cardiac Aging in Model Systems. Circ Res 118, 1553-1562 (2016). 
788 7. Birks, E. J. Molecular changes after left ventricular assist device support for heart failure. Circ. 789 Res. 113, 777-791 (2013).

790 8. Van Berlo, J. H. et al. C-kit+ cells minimally contribute cardiomyocytes to the heart. Nature 509, $791 \quad 337-341(2014)$.

792 9. Cho, S., Irianto, J. \& Discher, D. E. Mechanosensing by the nucleus: From pathways to scaling relationships. J Cell Biol 216, 305-315 (2017).

10. Janota, C. S., Calero-Cuenca, F. J. \& Gomes, E. R. The role of the cell nucleus in mechanotransduction. Current Opinion in Cell Biology vol. 63 204-211 (2020).

12. Khatau, S. B. et al. A perinuclear actin cap regulates nuclear shape. Proc. Natl. Acad. Sci. U. S. A. 106, 19017-19022 (2009).

13. Ramdas, N. M. \& Shivashankar, G. V. Cytoskeletal Control of Nuclear Morphology and Chromatin Organization. J. Mol. Biol. 427, 695-706 (2015).

14. Stephens, A. D., Banigan, E. J., Adam, S. A., Goldman, R. D. \& Marko, J. F. Chromatin and

15. Stephens, A. D. et al. Chromatin histone modifications and rigidity affect nuclear morphology

16. van Steensel, B. \& Belmont, A. S. Lamina-Associated Domains: Links with Chromosome Architecture, Heterochromatin, and Gene Repression. Cell 169, 780-791 (2017).

17. Dahl, K. N., Ribeiro, A. J. \& Lammerding, J. Nuclear shape, mechanics, and mechanotransduction. Circ Res 102, 1307-1318 (2008).

18. Chatzifrangkeskou, M., Kah, D., Lange, J. R., Goldmann, W. H. \& Muchir, A. Mutated lamin A modulates stiffness in muscle cells. Biochem. Biophys. Res. Commun. (2020) doi:10.1016/j.bbrc.2020.05.102.

19. Verstraeten, V. L. R. M., Ji, J. Y., Cummings, K. S., Lee, R. T. \& Lammerding, J. Increased mechanosensitivity and nuclear stiffness in Hutchinson-Gilford progeria cells: effects of farnesyltransferase inhibitors. Aging Cell 7, 383-393 (2008).

20. Lammerding, J. et al. Lamins a and C but not lamin B1 regulate nuclear mechanics. J. Biol. Chem. 281, 25768-25780 (2006).

21. Srivastava, L. K., Ju, Z., Ghagre, A. \& Ehrlicher, A. J. Spatial distribution of lamin A determines nuclear stiffness and stress-mediated deformation. bioRxiv 1-15 (2019) doi:10.1101/765263. 
822 22. Buxboim, A. et al. Matrix elasticity regulates lamin-A,C phosphorylation and turnover with feedback to actomyosin. Curr. Biol. 24, 1909-1917 (2014).

824 23. Dahl, K. N. et al. Distinct structural and mechanical properties of the nuclear lamina in Hutchinson-Gilford progeria syndrome. Proc. Natl. Acad. Sci. U. S. A. 103, 10271-10276 (2006).

24. Brandt, A. et al. Developmental control of nuclear size and shape by kugelkern and kurzkern. Curr. Biol. 16, 543-552 (2006).

25. Jevtić, P. et al. Concentration-dependent effects of nuclear lamins on nuclear size in xenopus and mammalian cells. J. Biol. Chem. 290, 27557-27571 (2015).

26. Pajerowski, J. D., Dahl, K. N., Zhong, F. L., Sammak, P. J. \& Discher, D. E. Physical plasticity of the nucleus in stem cell differentiation. Proc. Natl. Acad. Sci. U. S. A. 104, 15619-15624 (2007).

27. Bonne, G. et al. Mutations in the gene encoding lamin A/C cause autosomal dominant EmeryDreifuss muscular dystrophy. Nat. Genet. 21, 285-288 (1999).

28. Di Barletta, M. R. et al. Different mutations in the LMNA gene cause autosomal dominant autosomal recessive Emery-Dreifuss muscular dystrophy. Am. J. Hum. Genet. 66, 1407-1412 (2000).

29. R.G, T. M. et al. Natural history of dilated cardiomyopathy due to lamin A/C gene mutations. J.

Am. Coll. Cardiol. 41, 771-780 (2003).

30. Capell, B. C., Collins, F. S. \& Nabel, E. G. Mechanisms of cardiovascular disease in accelerated aging syndromes. Circ Res 101, 13-26 (2007).

31. Scaffidi, P. \& Misteli, T. Reversal of the cellular phenotype in the premature aging disease Hutchinson-Gilford progeria syndrome. Nat. Med. 11, 440-445 (2005).

32. Liu, B. et al. Genomic instability in laminopathy-based premature aging. Nat. Med. 11, 780-785 (2005).

33. Goldman, R. D. et al. Accumulation of mutant lamin A progressive changes in nuclear architecture in Hutchinson-Gilford progeria syndrome. Proc. Natl. Acad. Sci. U. S. A. 101, 89638968 (2004).

34. Shumaker, D. K. et al. Mutant nuclear lamin A leads to progressive alterations of epigenetic control in premature aging. Proc. Natl. Acad. Sci. 103, 8703 (2006).

35. Haithcock, E. et al. Age-related changes of nuclear architecture in Caenorhabditis elegans. Proc Natl Acad Sci U S A 102, 16690-16695 (2005).

36. Brandt, A., Krohne, G. \& Großhans, J. The farnesylated nuclear proteins KUGELKERN and LAMIN B promote aging-like phenotypes in Drosophila flies. Aging Cell 7, 541-551 (2008).

37. Scaffidi, P. \& Misteli, T. Lamin A-dependent nuclear defects in human aging. Science (80-. ). 312, 1059-1063 (2006). 
856 38. Larson, K. et al. Heterochromatin formation promotes longevity and represses ribosomal RNA synthesis. PLoS Genet. 8, (2012).

39. McClintock, D. et al. The Mutant Form of Lamin A that Causes Hutchinson-Gilford Progeria Is a Biomarker of Cellular Aging in Human Skin. PLoS One 2, e1269 (2007).

40. Messner, M. et al. Upregulation of the aging related LMNA splice variant progerin in dilated cardiomyopathy. PLoS One 13, e0196739 (2018).

41. Afilalo, J. et al. Age-related changes in lamin A/C expression in cardiomyocytes. Am. J. Physiol. Circ. Physiol. 293, H1451-H1456 (2007).

42. Freund, A., Laberge, R. M., Demaria, M. \& Campisi, J. Lamin B1 loss is a senescenceassociated biomarker. Mol. Biol. Cell 23, 2066-2075 (2012).

43. Chen, H., Zheng, X. \& Zheng, Y. Age-associated loss of lamin-b leads to systemic inflammation and gut hyperplasia. Cell 159, 829-843 (2014).

44. Han, L. et al. Lamin B2 Levels Regulate Polyploidization of Cardiomyocyte Nuclei and Myocardial Regeneration. Dev Cell (2020) doi:10.1016/j.devcel.2020.01.030.

45. Nikolova, V. et al. Defects in nuclear structure and function promote dilated cardiomyopathy in lamin A/C-deficient mice. J. Clin. Invest. 113, 357-369 (2004).

46. Cammarato, A. et al. A mighty small heart: the cardiac proteome of adult Drosophila melanogaster. PLoS One 6, e18497 (2011).

47. Nishimura, M. et al. A dual role for integrin-linked kinase and $\beta 1$-integrin in modulating cardiac aging. Aging Cell 13, 431-440 (2014).

48. Nava, M. M. et al. Heterochromatin-Driven Nuclear Softening Protects the Genome against Mechanical Stress-Induced Damage. Cell (2020) doi:10.1016/j.cell.2020.03.052.

49. Prakash, A. et al. Cardiac abnormalities in patients with hutchinson-gilford progeria syndrome. JAMA Cardiol. 3, 326-334 (2018).

50. Choi, H. M. T. et al. Third-generation in situ hybridization chain reaction: Multiplexed, quantitative, sensitive, versatile, robust. Dev. 145, 1-10 (2018).

51. bin Imtiaz, M. K. et al. Declining lamin B1 expression mediates age-dependent decreases of hippocampal stem cell activity. Cell Stem Cell 28, 967-977.e8 (2021).

52. Vogler, G. \& Ocorr, K. Visualizing the beating heart in Drosophila. J. Vis. Exp. 1425 (2009) doi:10.3791/1425.

53. Salick, M. R. et al. The scanning gradient Fourier transform (SGFT) method for assessing sarcomere organization and alignment. J. Appl. Phys. 127, 194701 (2020).

54. Cheedipudi Sirisha, M. et al. Genomic Reorganization of Lamin-Associated Domains in Cardiac Myocytes Is Associated With Differential Gene Expression and DNA Methylation in Human 
Dilated Cardiomyopathy. Circ Res 124, 1198-1213 (2019).

891 55. Davidson, E. H. \& Erwin, D. H. Gene Regulatory Networks and the Evolution of Animal Body $892 \quad$ Plans. Science (80-. ). 311, 796 LP - 800 (2006).

893 56. Dreesen, O. et al. Lamin B1 fluctuations have differential effects on cellular proliferation and 894 senescence. J. Cell Biol. 200, 605-617 (2013).

895 57. Schulze, S. R. et al. A comparative study of Drosophila and human A-type lamins. PLoS One 4, 896 (2009).

897 58. Cosgrove, B. D. et al. Nuclear envelope wrinkling predicts mesenchymal progenitor cell

59. Nava, M. M. et al. Heterochromatin-Driven Nuclear Softening Protects the Genome against

61. van Steensel, B. \& Belmont, A. S. Lamina-Associated Domains: Links with Chromosome

62. Zheng, X. et al. Lamins Organize the Global Three-Dimensional Genome from the Nuclear Periphery. Mol. Cell 71, 802-815.e7 (2018).

63. Hu, B. et al. Plant lamin-like proteins mediate chromatin tethering at the nuclear periphery. Genome Biol. 20, 1-18 (2019).

64. Ulianov, S. V et al. Nuclear lamina integrity is required for proper spatial organization of chromatin in Drosophila. Nat Commun 10, 1176 (2019).

65. Sawh, A. N. et al. Lamina-Dependent Stretching and Unconventional Chromosome Compartments in Early C. elegans Embryos. Mol. Cell 78, 96-111.e6 (2020).

66. Chang, L. et al. Nuclear peripheral chromatin-lamin B1 interaction is required for global integrity of chromatin architecture and dynamics in human cells. Protein Cell (2020) doi:10.1007/s13238020-00794-8.

67. Noguchi, A. et al. Decreased Lamin B1 Levels Affect Gene Positioning and Expression in Postmitotic Neurons. Neurosci. Res. (2021) doi:https://doi.org/10.1016/j.neures.2021.05.011.

68. Li, C.-L. et al. Region-specific H3K9me3 gain in aged somatic tissues in Caenorhabditis elegans. PLOS Genet. 17, e1009432 (2021).

69. Han, Z. \& Olson, E. N. Hand is a direct target of Tinman and GATA factors during Drosophila cardiogenesis and hematopoiesis. Development 132, 3525-3536 (2005). 
924 70. Bodyak, N. Gene expression profiling of the aging mouse cardiac myocytes. Nucleic Acids Res.

$925 \quad 30,3788-3794$ (2002).

926 71. Shen, T. et al. Tbx20 regulates a genetic program essential to adult mouse cardiomyocyte 927 function. J. Clin. Invest. 121, 4640-4654 (2011).

928 72. Sakabe, N. J. et al. Dual transcriptional activator and repressor roles of TBX20 regulate adult 929 cardiac structure and function. Hum. Mol. Genet. 21, 2194-2204 (2012).

930 73. Stennard, F. A. et al. Murine T-box transcription factor Tbx20 acts as a repressor during heart development, and is essential for adult heart integrity, function and adaptation. Development 132, 2451-2462 (2005).

74. Akazawa, H. \& Komuro, I. Roles of cardiac transcription factors in cardiac hypertrophy. Circ. Res. 92, 1079-1088 (2003).

76. Natarajan, A. et al. Human eHAND, but not dHAND, is down-regulated in cardiomyopathies. J. Mol. Cell. Cardiol. 33, 1607-1614 (2001).

77. Ahn, K. et al. Selection of internal reference genes for SYBR green qRT-PCR studies of rhesus monkey (Macaca mulatta) tissues. BMC Mol. Biol. 9, 1-8 (2008).

78. Ruiz-Villalba, A. et al. Reference genes for gene expression studies in the mouse heart. Sci.

Rep. 7, 1-9(2017).

944 79. Hertz, H. Ueber den kontakt elastischer koerper. J. fuer die Reine Angew. Math. 92, 156 (1881).

945 80. Smith, M. B., Chaigne, A. \& Paluch, E. K. An active contour ImageJ plugin to monitor daughter cell size in 3D during cytokinesis. Methods Cell Biol 137, 323-340 (2017).

81. Beri, P. et al. Cell adhesiveness serves as a biophysical marker for metastatic potential. Cancer Res. canres.1794.2019 (2019) doi:10.1158/0008-5472.CAN-19-1794.

82. Ocorr, K., Fink, M., Cammarato, A., Bernstein, S. \& Bodmer, R. Semi-automated Optical Heartbeat Analysis of small hearts. J. Vis. Exp. 1435 (2009) doi:10.3791/1435.

83. Martin, M. Cutadapt removes adapter sequences from high-throughput sequencing reads. EMBnet.journal 17, 10-12 (2011).

84. Babraham Bioinformatics - FastQC A Quality Control tool for High Throughput Sequence Data. https://www.bioinformatics.babraham.ac.uk/projects/fastqc/.

85. Dobin, A. et al. STAR: ultrafast universal RNA-seq aligner. Bioinformatics 29, 15-21 (2013).

86. S, A., PT, P. \& W, H. HTSeq--a Python framework to work with high-throughput sequencing data. Bioinformatics 31, 166-169 (2015). 
958 87. Love, M. I., Huber, W. \& Anders, S. Moderated estimation of fold change and dispersion for 959 RNA-seq data with DESeq2. Genome Biol. 20141512 15, 1-21 (2014).

960 88. L, W., S, W. \& W, L. RSeQC: quality control of RNA-seq experiments. Bioinformatics 28, 2184$2185(2012)$.

962 89. CRAN - Package fpc. https://cran.r-project.org/web/packages/fpc/index.html.

963 90. Bioconductor - topGO. https://bioconductor.org/packages/release/bioc/html/topGO.html.

964 91. Mitchell, A. L. et al. InterPro in 2019: improving coverage, classification and access to protein 965 sequence annotations. Nucleic Acids Res. 47, D351-D360 (2019).

966 92. LY, G. et al. The NCBI BioSystems database. Nucleic Acids Res. 38, (2010).

967 93. Subramanian, A. et al. Gene set enrichment analysis: A knowledge-based approach for 968 interpreting genome-wide expression profiles. Proc. Natl. Acad. Sci. 102, 15545-15550 (2005).

969 94. Liberzon, A. et al. Molecular signatures database (MSigDB) 3.0. Bioinformatics 27, 1739 (2011).

970 95. A, F. et al. The Reactome Pathway Knowledgebase. Nucleic Acids Res. 46, D649-D655 (2018).

971 96. DN, S. et al. WikiPathways: a multifaceted pathway database bridging metabolomics to other omics research. Nucleic Acids Res. 46, D661-D667 (2018).

973

974

975 


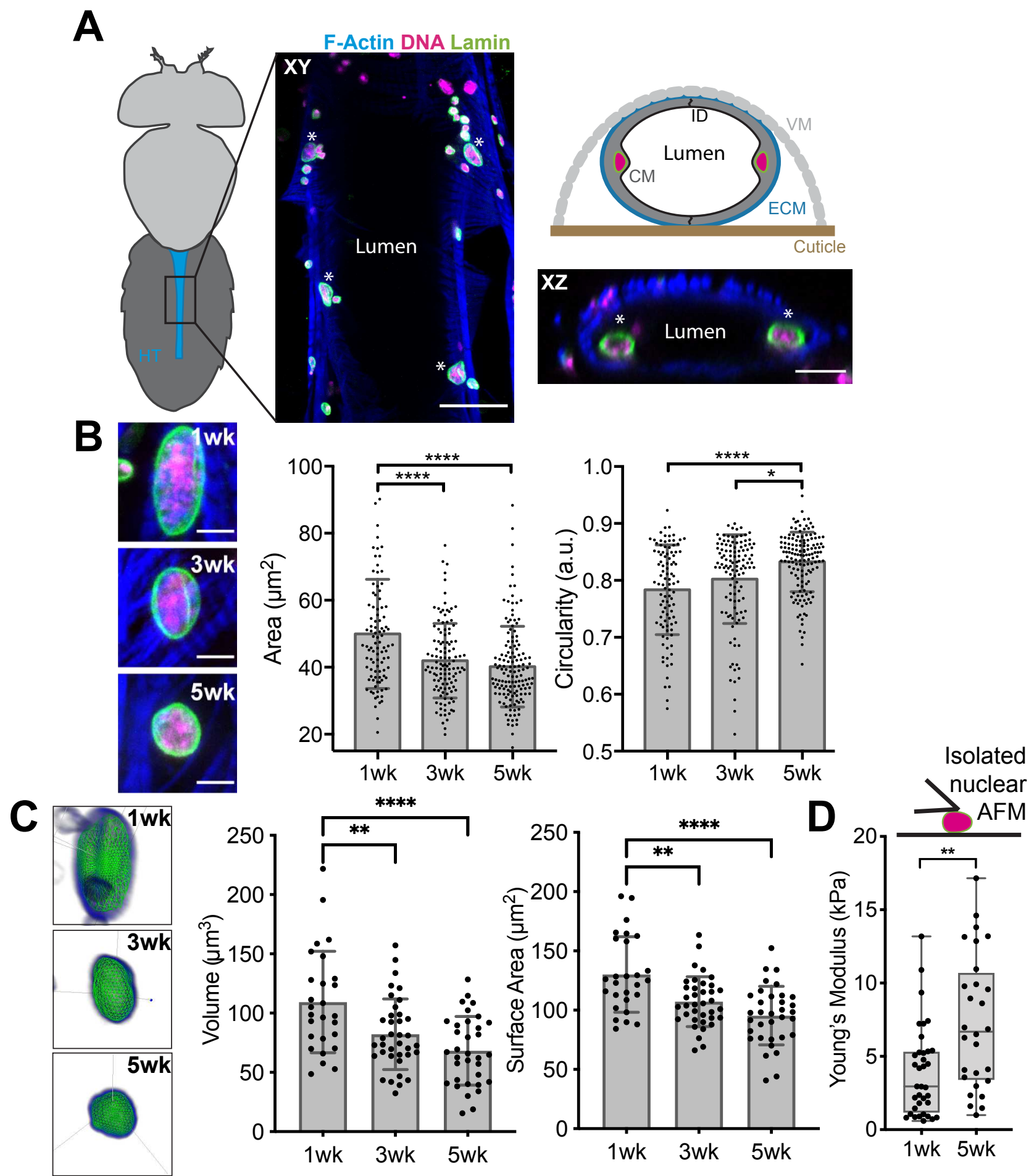

978 Figure 1: Age-Associated Changes in Cardiac Nuclear Morphology and Mechanics. (A)

979 Schematic of ventral Drosophila body plan with the heart tube in the abdomen highlighted in 980 blue. Expanded view of the heart tube shows a coronal (XY) confocal section through the heart 
981 tube (center) as well as a transverse (XZ) confocal section and schematic to highlight nuclear 982 position in the luminal space (right). Asterisks indicate cardiomyocyte nuclei. Scale bar is $20 \mu \mathrm{m}$. 983 (B) Images of $w^{1118}$ fly nuclei (left) and plot of their corresponding 2D projection data (right). 984 Scale bar is $5 \mu \mathrm{m} . \mathrm{n}=96,116$, and 141 nuclei for 1-, 3-, and 5-week adults, respectively. (C) 985 3D renderings of cardiac nuclei (left) and their corresponding for volume and surface area. $n=$ 986 27, 37, and 34 nuclei for 1- , 3-, and 5-week adult $w^{1118}$ flies respectively. (D) Atomic force 987 microscopy (AFM) nuclear indentation schematic (top) and plot of stiffness values, i.e., Young's 988 modulus, for nuclei of $w^{1118}$ flies (bottom). $\mathrm{n}=35$ and 28 nuclei for 1 - and 5-week adults, 989 respectively. ${ }^{* *} p<10^{-2}$ and ${ }^{* * *} p<10^{-4}$ by one-way ANOVA with Tukey multiple comparisons test 990 in (B-C) and unpaired t-test with Welch's correction in (D).

991

992 

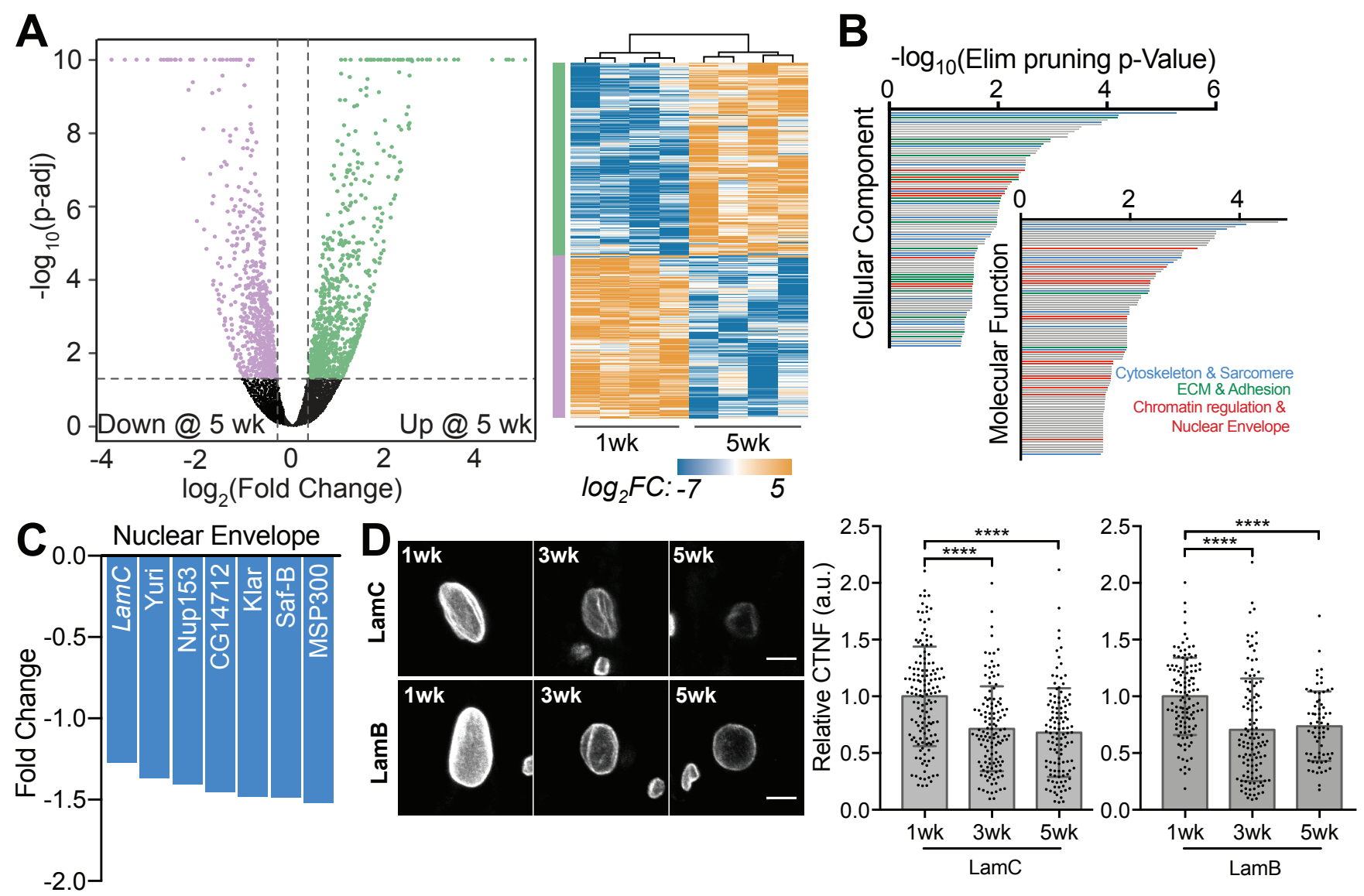

994 Figure 2: Natural Aging Downregulates Nuclear Envelope Proteins. (A) Volcano plot and 995 heat map of bulk RNA-seq from surgically dissected heart tubes. Fold change (FC) represents 996 5-week $w^{1118}$ fly hearts normalized to 1-week old hearts and p-adjusted was computed from 997 quadruplicate repeats. 1,268 differentially expressed genes (DEGs) were assessed from cutoffs 998 of $-1.25>$ FC $>1.25$ and $\mathrm{p}$-adj < 0.05 (dashed lines) from comparisons of 15 fly hearts in 999 quadruplicate biological replicates; DEGs increasing and decreasing with age are shown in 1000 green and purple, respectively. (B) The top 100 molecular function and cellular component gene 1001 ontological terms are plotted based on elimination pruning p-value. Terms related to cytoskeleton 1002 \& sarcomere, ECM \& adhesion, and chromatin remodeling \& nuclear envelope are annotated by 1003 color. (C) Expression of selected genes associated with nuclear envelope terms are plotted, with 1004 Lamin C (LamC) expression noted with italics. (D) Confocal projection images of cardiomyocyte 1005 nuclei showing Lamin B (LamB) and C expression with age (left). Scale bar is $5 \mu \mathrm{m}$. (right) 1006 Corrected total nuclear fluorescence (CTNF), which adjusts for nucleus size, is plotted for LamB 1007 and $\operatorname{LamC}$ as a function of adult fly age. $\mathrm{n}=141,124,116,114,107$, and 69 nuclei/condition 
1008 from left to right for LamC and LamB aged 1-, 3-, or 5-weeks of adulthood, respectively. ${ }^{* * *} \mathrm{p}<10^{-}$ $1009{ }^{4}$ by one-way ANOVA with Tukey multiple comparisons test.

1010

1011 


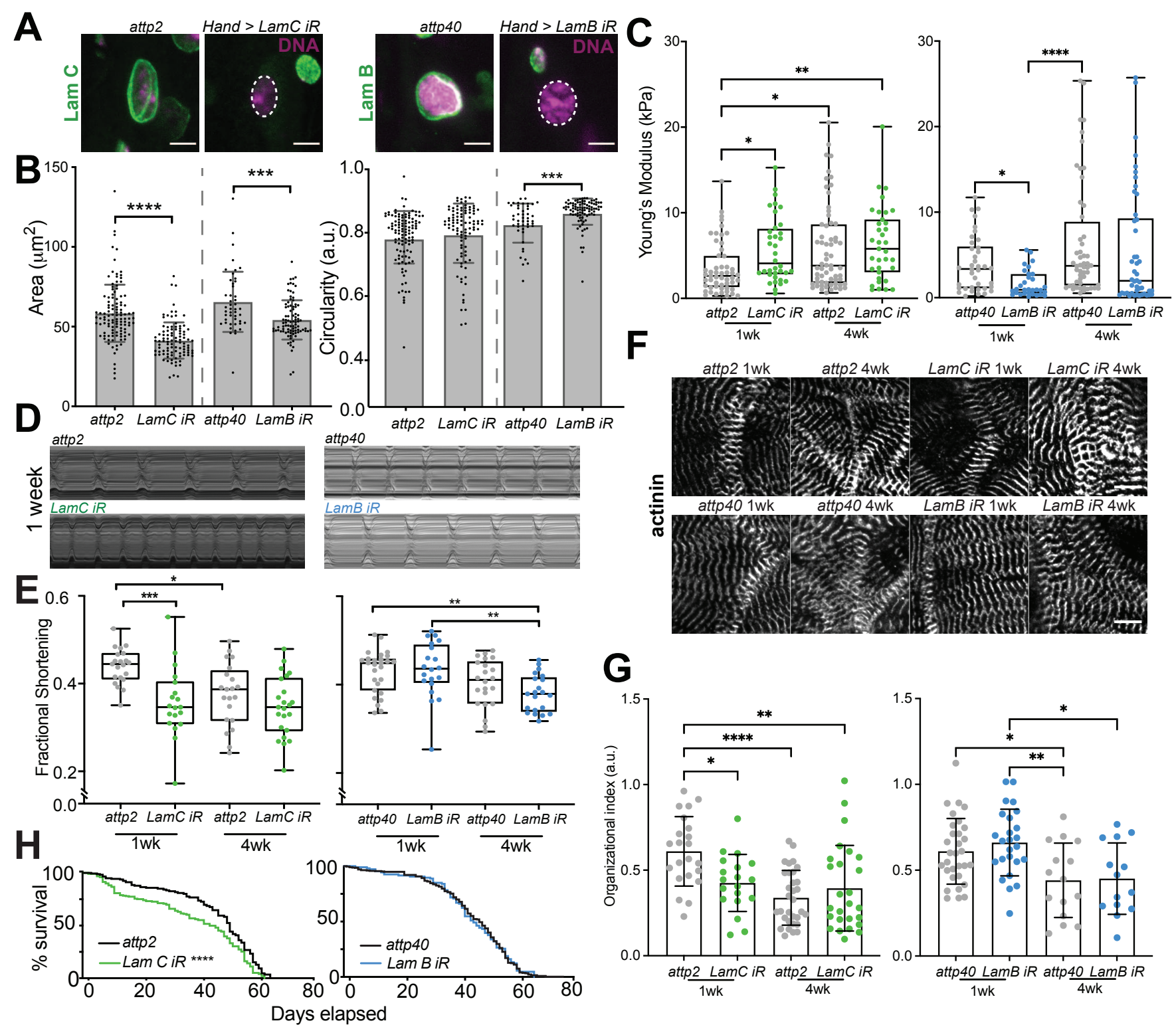

1013 Figure 3: LamC, but not LamB, impacts Cardiomyocyte Aging, Heart Function, and

1014 Lifespan. (A) Confocal cross-section images are shown for transgenic flies knocking down 1015 LamB and LamC by RNAi (right) and their background fly line (left). Dashed lines indicate nuclear 1016 position based on DNA. Scale bar is $5 \mu \mathrm{m}$. (B) Plots quantifying nuclear area (left) and circularity 1017 (right) based on confocal images of LamB and LamC RNAi lines and their genetic control 1018 background at 1-week of adulthood. $n=111,95,46$, and 90 (hearts/condition; left to right). (C) 1019 Plot of Young's modulus values is shown for nuclei of the indicated adult ages for LamC (green), $1020 \mathrm{LamB}$ (blue) $R N A i$, and their control strains (grey). $\mathrm{n}=53,36,63,35,38,31,52$, and 46 1021 (hearts/condition; left to right). (D) Representative kymographs of surgically exposed heart tubes 
1022 for $\operatorname{LamC}$ (green), LamB (blue) RNAi, and their control strains. (E) Fractional shortening at 11023 and 4-weeks of adulthood is plotted for $\operatorname{LamC}$ (green) and $\operatorname{LamB}$ (blue) RNAi, and their control 1024 strains. $n=21,18,21,23,27,20,22$, and 20 (hearts/condition; left to right). (F) Representative 1025 images of $\alpha$-actinin staining for the indicated transgenic flies and their control backgrounds 1026 (paired by row). Scale bar is $10 \mu \mathrm{m}$. (G) Organizational index is plotted for each heart tube. $\mathrm{n}=$ $102722,19,32,26,31,25,15$, and 14 (hearts/condition; left to right). (H) Kaplan-Meier survival curve 1028 for $\operatorname{LamC}$ (green) and $\operatorname{LamB}$ (blue) RNAi, and their control strains. 102, 148, 95, and 200 flies 1029 for attp2, LamC RNAi, attp40, and LamB RNAi, respectively, were used in the plot. ${ }^{*} \mathrm{p}<0.05$, $1030{ }^{* *} p<10^{-2},{ }^{* * *} p<10^{-3}$, and ${ }^{* * * *} p<10^{-4}$ by one-way ANOVA with Tukey multiple comparisons test for 1031 (B-G). ${ }^{* * *} p<10^{-4}$ based on Log-Rank (Mantel-Cox) test in $(H)$. 

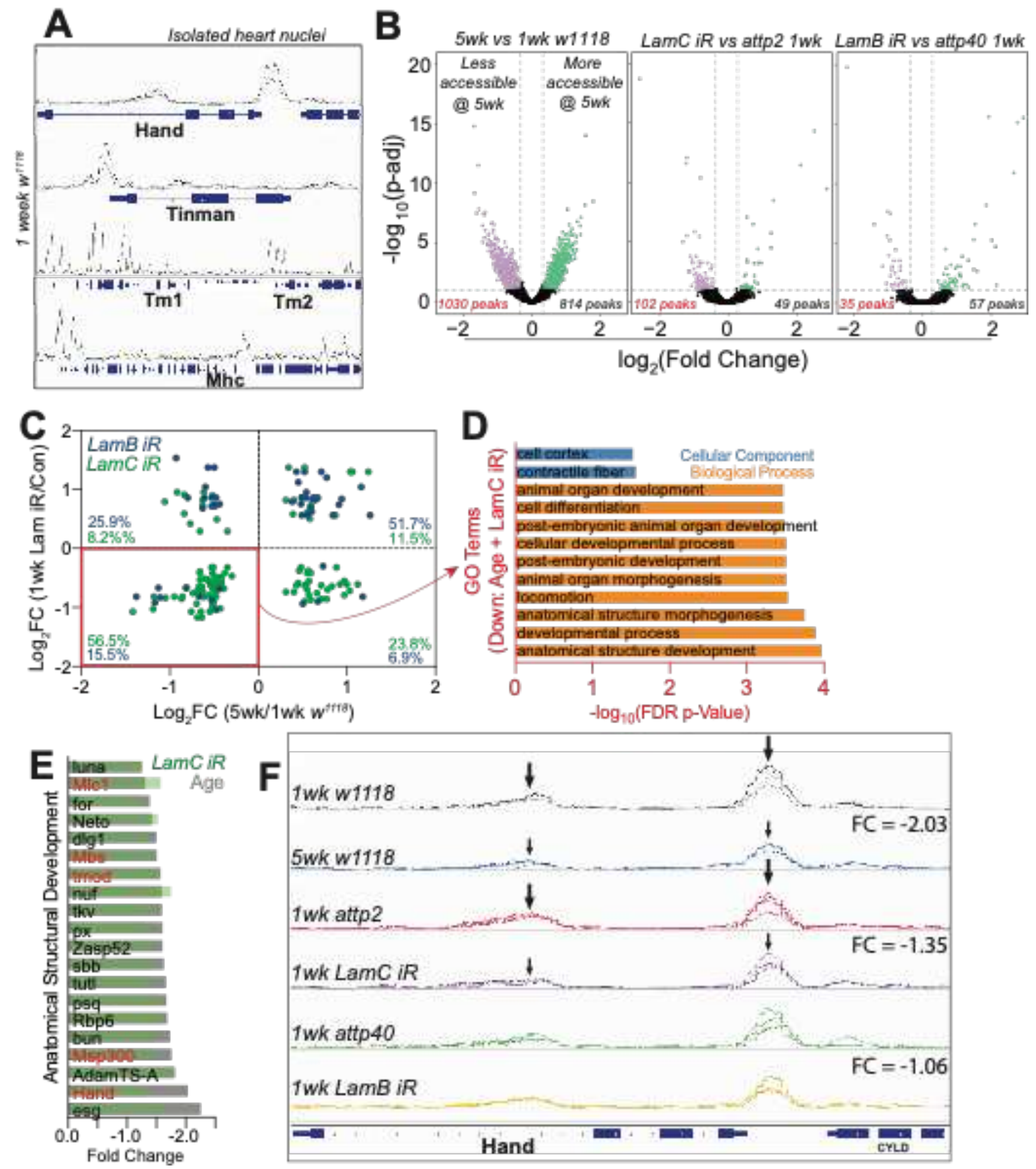

Figure 4: Chromatin Accessibility Decreases with Age and LamC RNAi at Sites of Myogenic Control. (A) Map of accessibility peaks for Drosophila cardiac transcription factor genes Hand (top) and tinman (tin; upper middle) and sarcomere genes Tropomyosin (Tm1 and 
1038 Tm2; lower middle) and Mhc (bottom). Data is shown in triplicate sequencing runs using 1-week 1039 adult $w^{1118}$ flies, i.e., 3 lines plotted in the panel. (B) Volcano plots of the indicated aging or 1040 transgenic comparisons of differentially accessible regions (DARs) from ATAC-seq. The number 1041 of peaks is annotated at the bottom for each comparison indicating if the region is more (black) 1042 or less (red) accessible relative to the comparator line. (C) Scatter plot is shown for ATAC-seq 1043 data comparing the fold change in accessibility for genes based on effects from aging and LamC 1044 (green) or LamB (blue) RNAi. Percentage of data in each quadrant are shown. (D) Top gene 1045 ontological terms are plotted for co-downregulated peaks (closest associated gene) in aged and 1046 LamC RNAi fly comparisons and ordered based on the false discovery rate p-value. (E) Genes 1047 within the anatomical structure term were plotted for their fold change for aging (gray) and LamC $1048 R N A i$ (green). Genes names in red represent myogenic transcription factors or muscle-specific 1049 structure proteins. (F) Map of accessibility peaks for the myogenic transcription factor Hand. 1050 Arrows indicate the location of a common DAR in Hand that is present and reduces in aged and 1051 LamC RNAi flies but not in LamB RNAi flies. Multiple lines per map indicate multiple sequencing 1052 runs of biological replicates. DAR fold change is annotated for each comparison. 

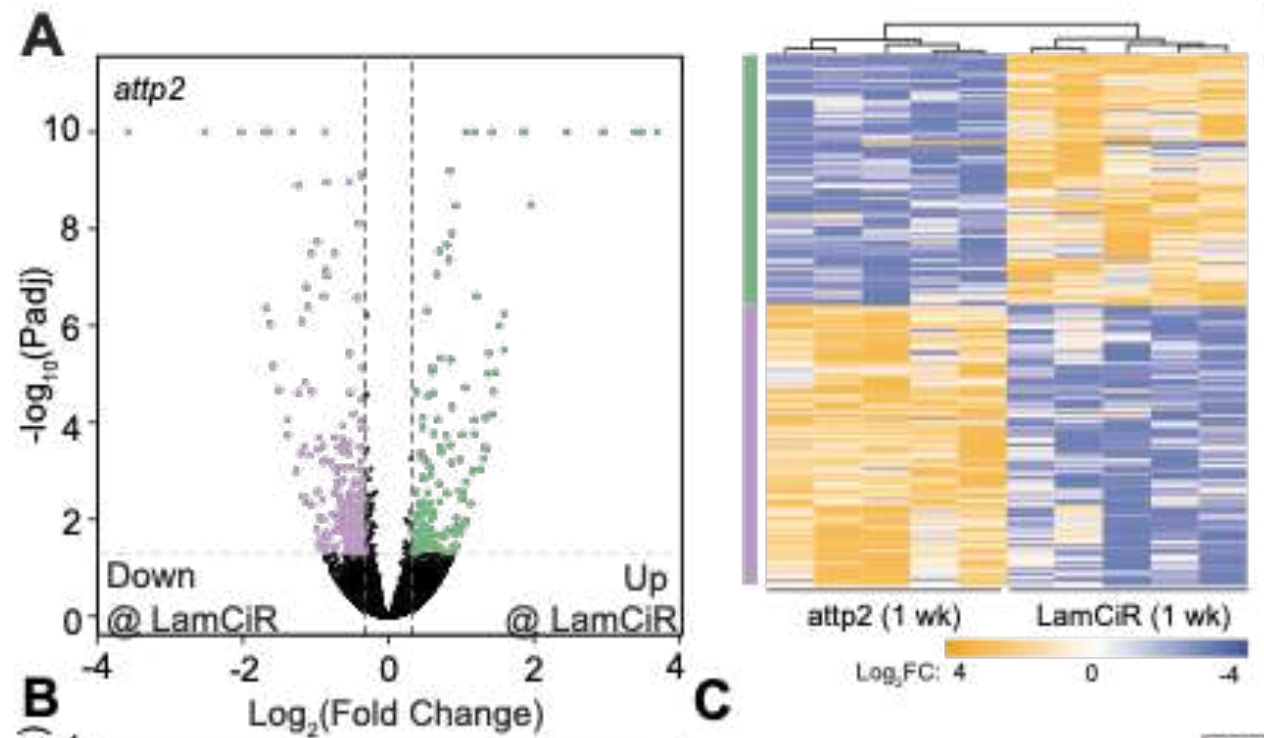

Anatomical Structure Development $\log _{2}$ (FC)
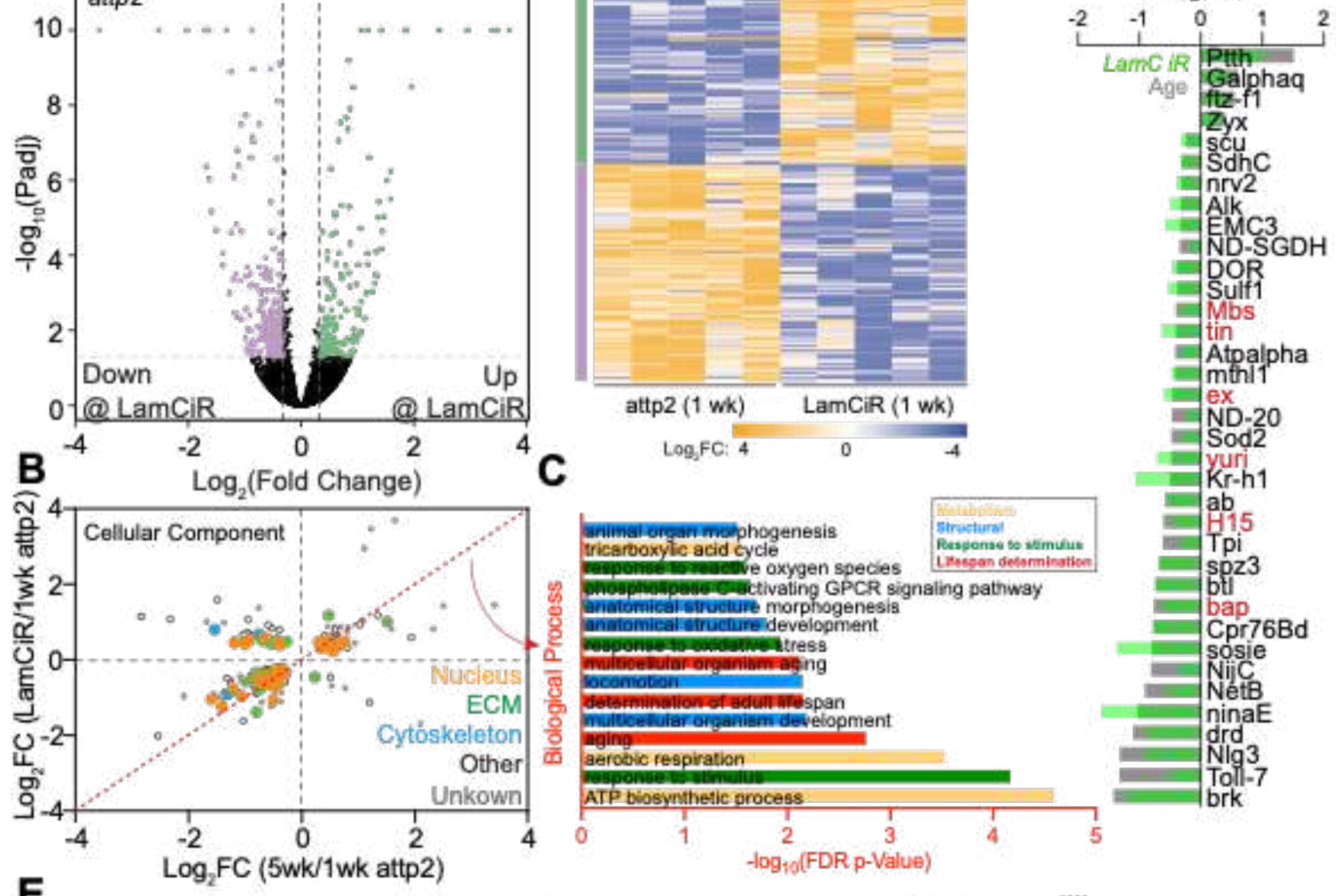

E
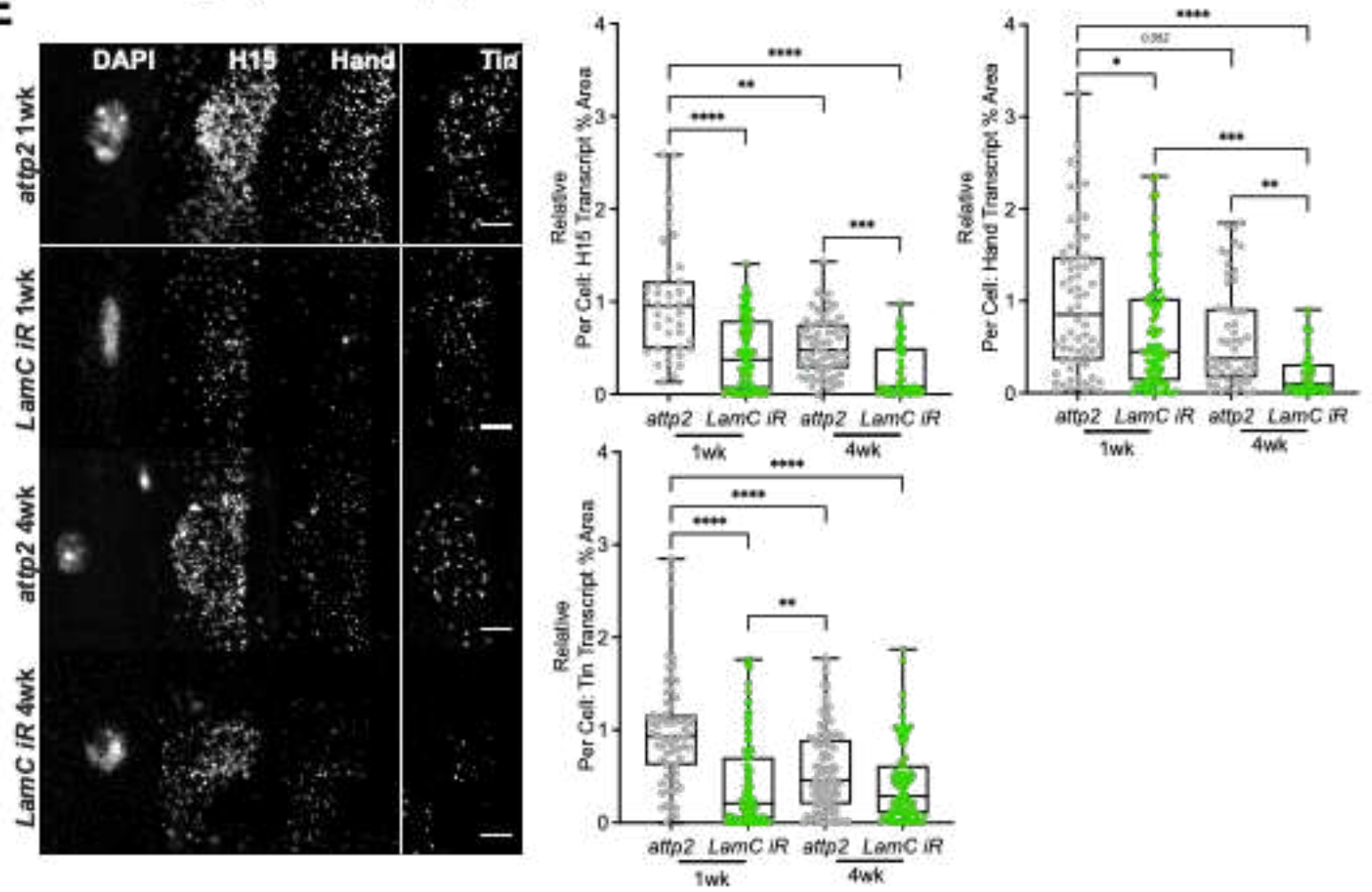
1056 Figure 5: LamC Loss Transcriptionally Regulates Myogenic Transcription Factors. (A) 1057 Volcano plot and heat map of bulk RNA-seq from surgically dissected heart tubes from LamC 1058 RNAi flies compared to attp2 control background flies at 1-week of age. 344 DEGs were 1059 assessed from cutoffs of $-1.25>F C>1.25$ and $p$-adj $<0.05$ (dashed lines) from comparisons of 106015 fly hearts in quadruplicate biological replicates; DEGs increasing and decreasing with LamC 1061 RNAi are shown in green and purple, respectfully. (B) Scatter plot is shown comparing fold 1062 changes from aging (1- and 5-weeks of age) and LamC RNAi compared to control background 1063 (attp2). Data was categorized based on which cellular component ontology term it most closely 1064 matches. Distance from the red dashed line of unity was used identify co-regulated genes whose 1065 (C) biological process ontology terms were annotated and ordered based on their false discovery 1066 rate p-value. (D) Genes within the anatomical structure term were plotted for their fold change 1067 for aging (gray) and LamC RNAi (green). Genes names in red represent myogenic transcription 1068 factors or muscle-specific structure proteins. (E) Representative images (left) of in situ 1069 hybridization chain reaction for transcription factors $\mathrm{H} 15$, Hand, and tin, co-stained with DAPI, 1070 for LamC RNAi and control attp2 flies at 1- and 4-weeks of adulthood. A plot for each transcription 1071 factor is also shown (right) and quantifies the per cell percent area covered by each transcript. 1072 For H15, $\mathrm{n}=39,76,64$, and 45 cells for 1-week control, 1-week LamC RNAi, 4-week control, 1073 and 4-week LamC RNAi, respectively. For Hand, $\mathrm{n}=71,84,52$, and 43 cells for 1 -week control, 1074 1-week LamC RNAi, 4-week control, and 4-week LamC RNAi, respectively. For $\operatorname{tin}, \mathrm{n}=69$, 101, 1075 103, and 85 cells for 1-week control, 1-week LamC RNAi, 4-week control, and 4-week LamC $1076 R N A i$, respectively. ${ }^{*} \mathrm{p}<0.05,{ }^{* *} \mathrm{p}<10^{-2},{ }^{* * *} \mathrm{p}<10^{-3}$, and ${ }^{* * * *} \mathrm{p}<10^{-4}$ by one-way ANOVA with Tukey 1077 multiple comparisons test.

1078 1079 

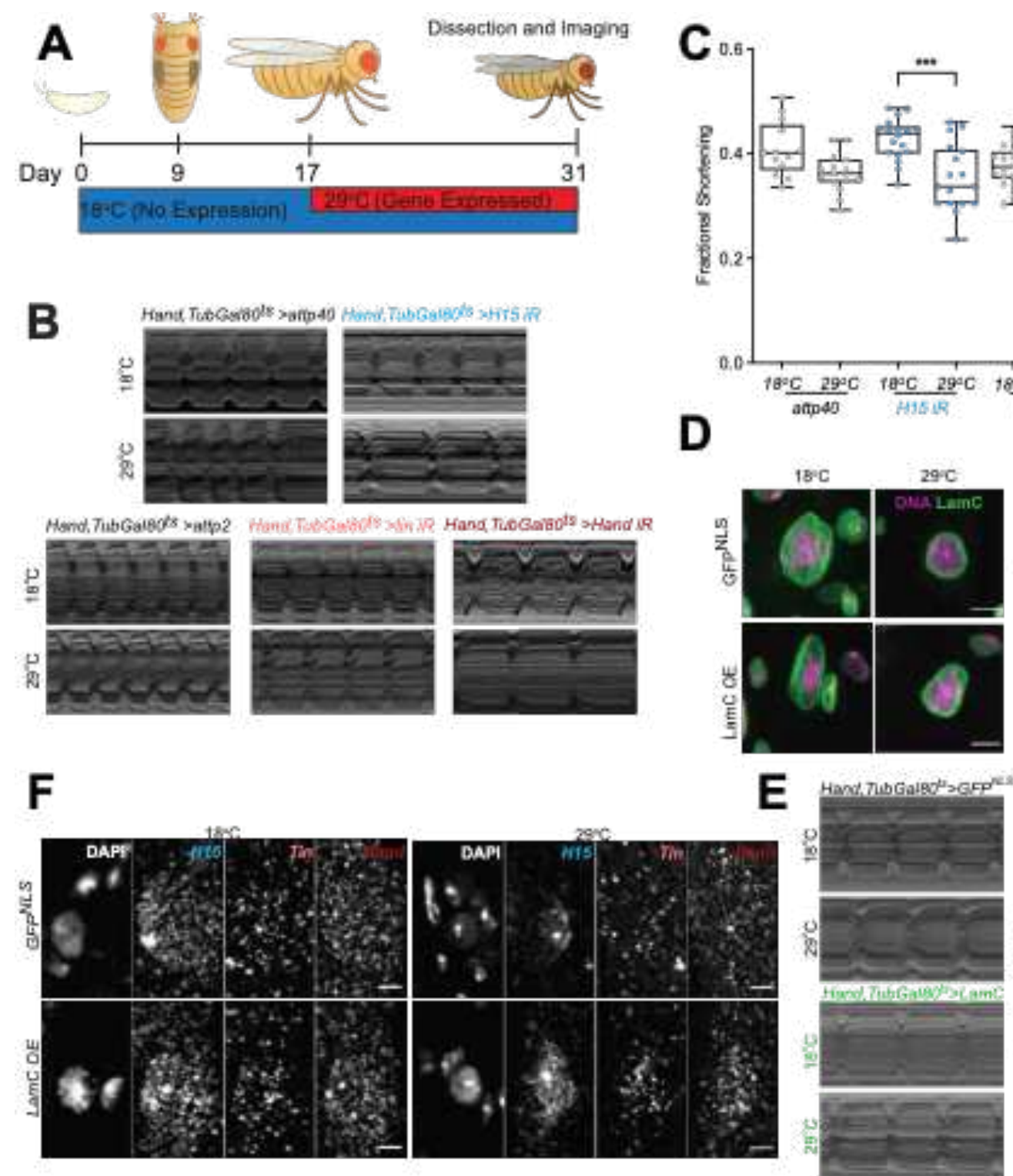

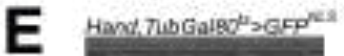
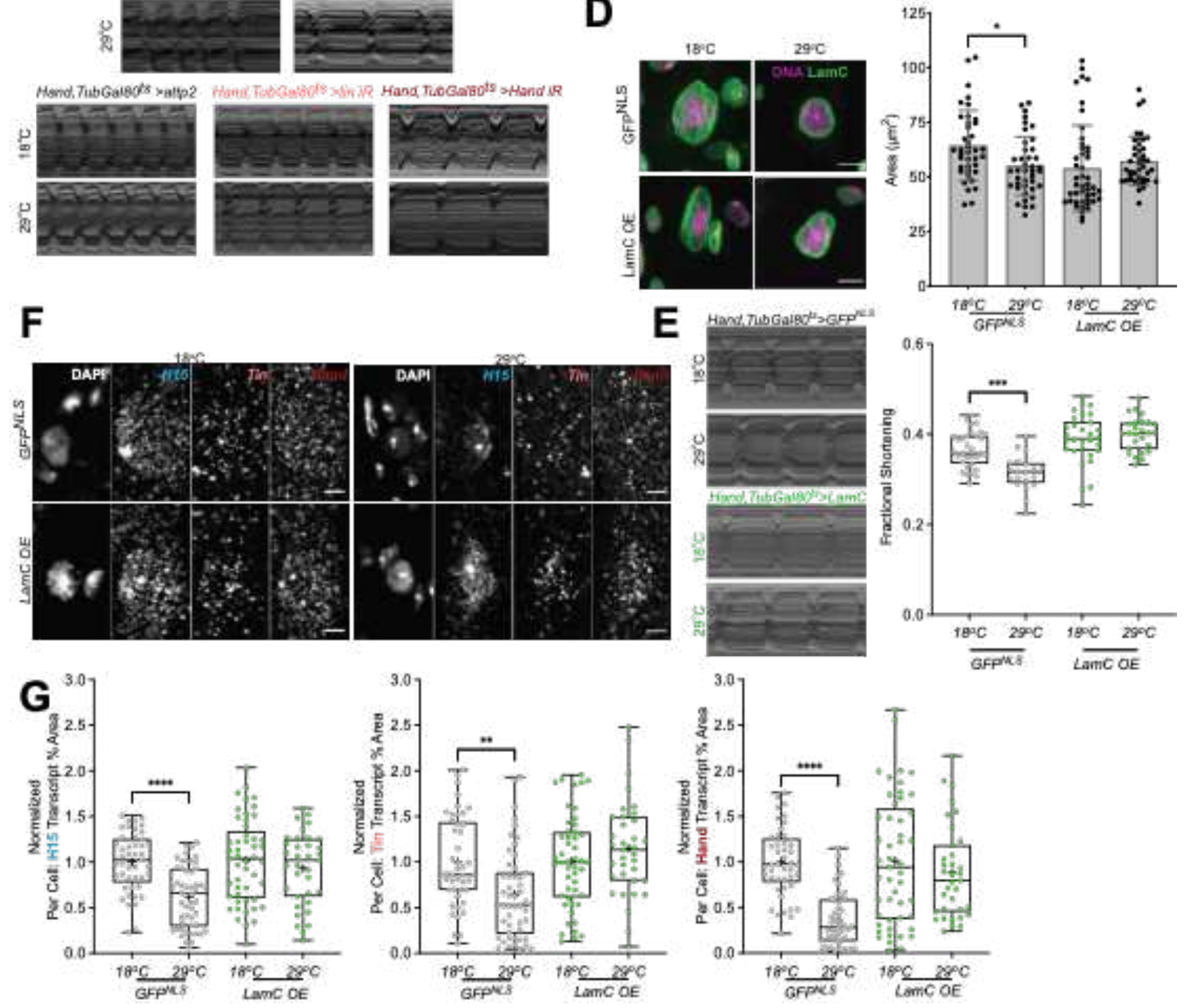

1081 Figure 6: Myogenic Transcription Factors Diminish with Age and LamC Overexpression 1082 can Rescue Decreased Heart Function. (A) Schematic of temperature sensitive transgenic 
1083 expression where $29^{\circ} \mathrm{C}$ enables transgenic expression due to the denaturation of Gal4 1084 transcription factor suppressor, Gal80 ${ }^{\text {ts }}$. (B) Representative kymographs of surgically exposed 1085 heart tubes at $18^{\circ} \mathrm{C}$ and $29^{\circ} \mathrm{C}$ for attp2 and attp40 controls (black), tin RNAi (pink), Hand RNAi 1086 (brown), and H15 RNAi (blue) and (C) quantification of their respective fractional shortenings at 1087 indicated temperatures are shown. $n=13,15,18,15,26,27,16,19,29,27,29$, and 21 (heart 1088 tubes/transgene/ temperature; left to right). (D) Representative images (left) of nuclei from flies 1089 at $18^{\circ} \mathrm{C}$ and $29^{\circ} \mathrm{C}$ expressing a generic GFP ${ }^{N L S}$ transgene or an overexpression of LamC (LamC 1090 OE). To the right is a plot of projected nuclear area as a function of temperature and transgene 1091 expression. $n=39,40,46$, and 41 nuclei (left to right). (E) Representative kymographs (left) of 1092 surgically exposed heart tubes for LamC OE (green) and generic GFPNLS transgene (black) at $109318^{\circ} \mathrm{C}$ and $29^{\circ} \mathrm{C}$. Fractional shortening (right) is plotted for the same conditions and transgenes. $1094 \mathrm{n}=37,20,30$, and 26 heart tubes. (F) For GFPNLS and $\operatorname{LamC} \mathrm{OE}$ at $18^{\circ} \mathrm{C}$ and $29^{\circ} \mathrm{C}$, 1095 representative images for in situ hybridization chain reaction are shown for tin (pink), Hand 1096 (brown), and H15 (blue) transcripts. (G) A plot for each transcription factor is shown and 1097 quantifies the per cell, percentage area for each transcript. ${ }^{*} p<0.05,{ }^{* *} p<10^{-2},{ }^{* * *} p<10^{-3}$, and $1098{ }^{* * * *} p<10^{-4}$ by one-way ANOVA with Kruskal-Wallis test and Dunn's comparisons test. 


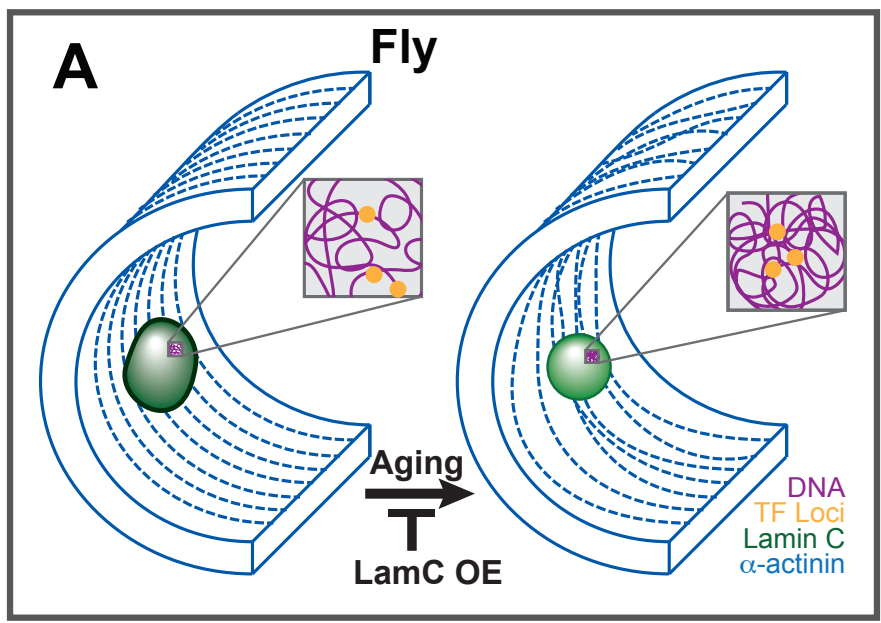

Mouse
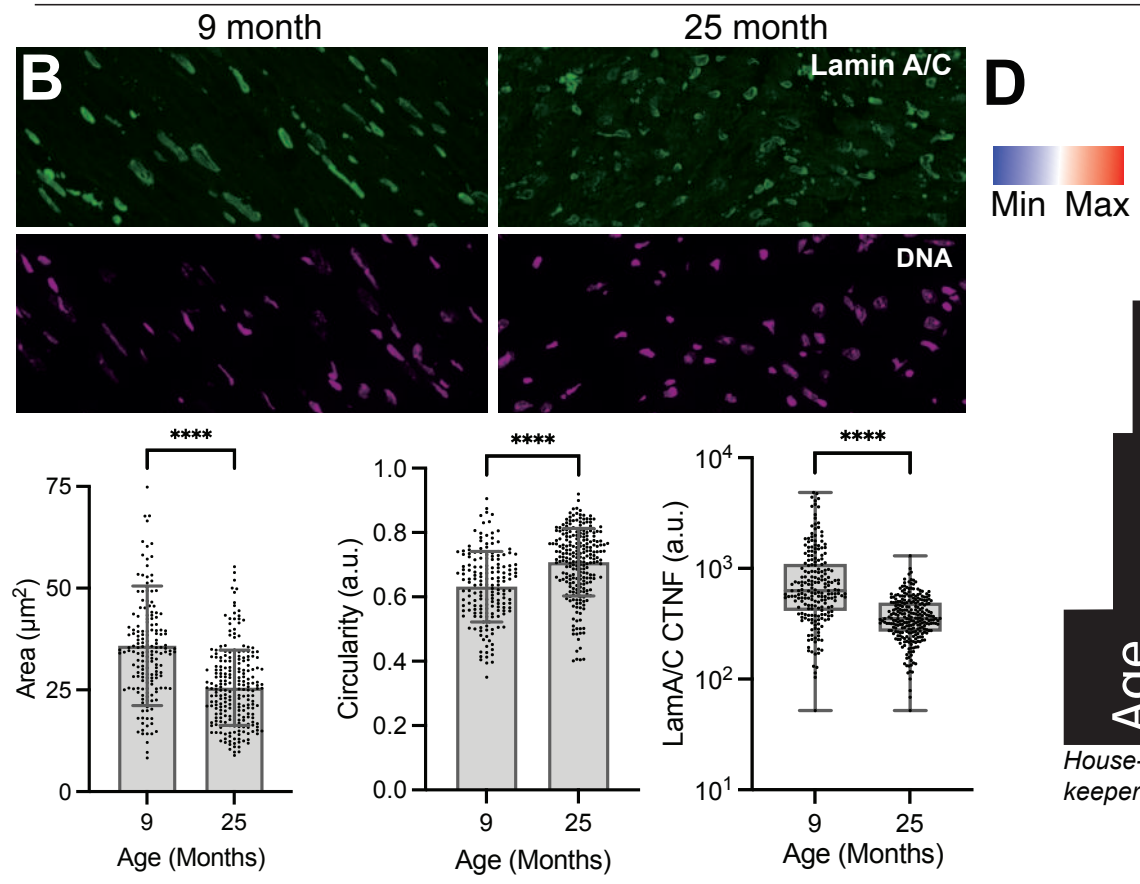

in Max

Monkey
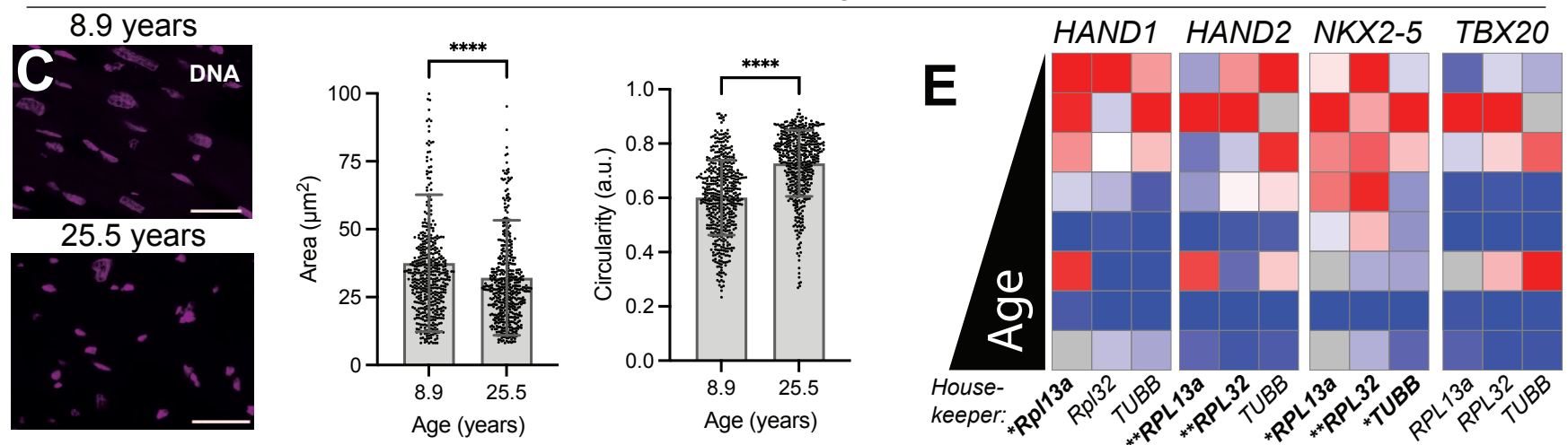

Figure 7: Nuclear remodeling induces adult-onset transcription factor loss, a process 
1103 conserved in Mice and Non-human Primates. (A) Schematic depicting how age-associated, 1104 cardiac-specific reduction of nuclear lamins reduces nuclear volume and chromatin accessibility, 1105 especially for myogenic transcription factors. With less muscle transcription from key cardiac loci, 1106 sarcomeres become disordered with age and heart function is reduced. Lamin overexpression 1107 can overcome age-associate reduction and preserve function. (B) Immunofluorescent staining 1108 of mouse heart sections at 9 and 29 months of age with anti-LamA/C (green) and DAPI 1109 (magenta). Below shows plots of projected nuclear area and circularity with respective ages. $n$ $1110=3$ biological replicates and 3 technical replicates. (C) Immunofluorescent staining of monkey 1111 left ventricle sections at 8.9 and 25.5 years of age with anti-LamA/C (green) and DAPI (magenta). 1112 Below shows plots of projected nuclear area and circularity with respective ages. $n=3$ biological 1113 replicates and 3 technical replicates. qPCR results are plotted for (D) mouse and (E) rhesus 1114 macaque for the myogenic transcription factors Hand 1, Hand 2, Nkx2-5, and TBX20 as 1115 normalized by housekeepers Eef1e1, Rpl4, and ACTB for mouse and Rpl13a, Rpl20, and 1116 TUBB2 for macaque. Data were normalized to maximum and minimum expression within each 1117 gene and housekeeper for heatmap. ${ }^{*} p<0.05$, ${ }^{* *} p<10^{-2}$, ${ }^{* * *} p<10^{-3}$, and ${ }^{* * *} p<10^{-4}$ by unpaired t1118 test (B, circularity) and with Welch's correction in (B, nuclear area). Significance in (C) and (E) 1119 indicate deviation from 0 for simple linear regression. 


\section{Supplementary Files}

This is a list of supplementary files associated with this preprint. Click to download.

- ManuscriptSupplementalNatureAging.pdf 